\title{
Information Overload in a Network of Targeted Communication
}

\author{
Timothy Van Zandt* \\ INSEAD
}

18 May 2001

\begin{abstract}
As the costs of generating and transmitting information fall, the main bottlenecks in communication networks are becoming the human receivers, who are overloaded with information. For networks of targeted communication, this paper discusses the meaning of information overload, provides a theoretical treatment of its causes, and examines mechanisms for allocating the attention of receivers. Mechanisms for allocating attention include surcharges on communication and auctions. These mechanisms increase the cost of sending messages and shift the task of screening messages from the receivers to the senders. This shift may benefit both the receivers and the senders because the senders know the contents of the messages whereas the receivers do not. We show that, if the communication cost is low, then an increase in the communication cost benefits most (but not all) receivers. The increase benefits all the senders if either the extra cost is a tax that is redistributed to them as lump-sum transfers or the senders information about the receivers is sufficiently accurate.
\end{abstract}

Author's address:

INSEAD

Boulevard de Constance 77305 Fontainebleau CEDEX France

$\begin{array}{ll}\text { Voice: } & +33160724981 \\ \text { Fax: } & +33160746192 \\ \text { Email: } & \text { tvz@econ.insead.edu } \\ \text { Web: } & \text { zandtwerk.insead.edu }\end{array}$

Voice: $\quad+33160724981$

Email: tvz@econ.insead.edu

Web: zandtwerk.insead.edu 


\section{Contents}

1 Introduction 1

2 Literature review 2

3 Model 4

4 Equilibrium $\quad 7$

5 Strategies that maximize the senders' total payoffs 9

6 Type-dependent mechanisms for allocating attention 11

7 Allocating attention through uniform surcharges $\quad 12$

8 Sales and payoffs when the communication cost is low 14

$9 \begin{array}{ll}\text { Receiver welfare and the cost of communication } & 17\end{array}$

10 Remarks $\quad 18$

A Demographic data and types $\quad 19$

$\begin{array}{lr}\text { B Proofs } & 19\end{array}$

References $\quad 25$ 


\section{Introduction}

The physical resources for communication, such as data networks and the postal service, are scarce. Attempts have always been made to allocate these resources efficiently through price mechanisms. However, human communication is not merely the transmission of bits from one computer to another; information must go from brain to brain. The cost of physical communication resources has fallen so much that the relatively scarce resource is now the human attention needed to process and understand information. For example, compare the cost of mailing a working paper to a colleague with the opportunity cost of the time it takes your colleague to read and understand your paper. Yet human attention is not priced in networks.

Should it be? Human attention is not a passive resource; rather, each person controls the allocation of her attention to the information she receives. Since information is freely disposable, the mere fact that people receive more information than they can process does not mean they receive too much.

Nevertheless, complaints about information overload are common. This paper provides an interpretation of information overload such that "nearly rational" decision makers can be made worse off by receiving more information. The decision makers are limited in their ability to process information and are forced to search among messages - without knowing their content, of course - if they receive more than they can process. An increase in the amount of information can be detrimental if it is accompanied by a decrease in the average value of the information. We construct a model of networked communication - messages are targeted to individual receivers based on imperfect demographic data - in which precisely this link between quantity and quality arises endogenously as the outcome of strategic interaction between senders; this link due to the fact that human attention is an unpriced resource.

Consider in more detail this last point. If there were only a single sender, then she would not choose to overload any receivers, preferring to decide herself which messages receivers process rather than having receivers do so blindly, just as a single sender would not overload an e-mail network to the point that messages are dropped. However, when there are multiple senders, information overload is an externality between the senders, as each sender's messages crowd out messages of other senders. Thus, inadequate screening by senders arises because the physical cost of generating and transmitting information no longer rations access to receivers' attention.

Our analysis is via a simple model in which there is a one-time, one-way flow of information from several senders to a large population of receivers, and in which communication is targeted in the sense that senders can send their messages to any subset of receivers. An example is the communication from firms to consumers via targeted advertising, such as direct mail, e-mail, and fax. Targeted communication contrasts with untargeted or broadcast communication; in the latter, messages are sent either to all receivers or to none. The web, billboards, and television are means of untargeted communication.

What differentiates our model theoretically from a standard commons game is that we consider a large population of unpriced resources - corresponding to the large population of receivers - and derive our main new insights from the aggregate properties of the equilibria and the relation to the imperfect marketing data of the senders. In our treatment of mechanisms for allocating attention, we do not restrict attention to ones that treat each receiver independently, decomposing the problem into many independent resource pricing problems. Such mechanisms, in which senders are charged a different cost for each receiver they target, are excessively complex to implement. Instead, we consider the effects of uniform changes in 
communication costs, either as exogenous technological parameters or as the result of taxes. Some of our main results are that, if transmission costs are low enough, then (a) the senders unanimously benefit from the imposition of a tax on their communication if the revenues are returned to them as lump-sum transfers and (b) if the senders' marketing data are accurate enough then they unanimously benefit from an increase in communication costs even if the extra costs are not reimbursed.

We began this introduction by discussing information overload as a complaint of receivers, yet we have shifted the point of view to that of the senders. One of the messages of this paper is precisely that information overload is a consequence of the strategic interaction among senders of messages and that they also are directly harmed by their collective overexploitation of the receivers' attention. To focus on this strategic interaction, we model the receivers passively and do not study the complex screening methods they might use to cope with information overload.

However, we can still draw conclusions from the model about the welfare properties of the equilibria from the point of view of the receivers. The interests of senders and receivers do not coincide perfectly, but they do coincide in part. A sender benefits from having a receiver process a message only if the message influences the actions of the receiver-and this is precisely when the message also benefits the receiver. Thus, when senders reduce their screening and send messages they believe unlikely to interest the receivers, the receivers find that they are searching among messages that are more numerous but of less interest on average. Using this link, we are able to show that, if the senders' transmission cost is low enough, then the aggregate welfare of receivers rises with this cost even though some receivers (those about whom the senders have inaccurate information) are better off with a lower communication cost. The message of this paper is supported by a recent laboratory experiment by Kraut et al. (2000), who found that charging for e-mail can increase the effectiveness of communication.

We begin the paper with a literature review in Section 2. Than a game with costly targeted communication is introduced in Section 3. In Section 4, we show that the game has an equilibrium and we illustrate the game with a few examples. In Section 5, we show that the total payoffs of the senders are often not maximized because senders communicate too much. We then consider mechanisms for alleviating this inefficiency: in Section 6 , we study pricing and bidding mechanisms that are tailored to each receiver type; in Section 7 , we consider whether efficiency can be achieved by using surcharges that are uniform across receivers. In Section 8, we examine how the payoffs of the senders vary with the cost of communication in a neighborhood of zero. The welfare of receivers is taken up in Section 9, followed by concluding remarks in Section 10. Appendix A describes a primitive model of receivers and of the information that senders have about them, which supports the reduced-form used in the body of the paper; Appendix B contains all the proofs.

\section{Literature review}

Research on information overload is scattered in the fields of computer and information science, marketing, law, psychology, and economics. The research found by the author has various objectives and various approaches, all distinct from those of this paper. Here is an incomplete review.

Researchers in information science are interested in developing computer-mediated communication systems to increase the efficiency of information screening by receivers in order to reduce the relative scarcity of attention in electronic networks. For example, Malone 
et al. (1987) and Lai et al. (1988) developed the so-called "Information Lens" and "Object Lens" systems.

In a brief essay, Denning (1982) called attention to the problem of information overload. He also suggested several ways of restricting communication in order to reduce information overload, such as setting up different paths for different types of mail, restricting access to mailboxes to specifically authorized users, setting up private mailboxes with unlisted addresses, and allocating attention by bids attached to each message, denominated in real or funny money. Only the bidding mechanism is discussed in this paper. Hiltz and Turoff (1985) counter that the task of screening should shift from senders to receivers, rather than in the opposite direction. However, their worry is primarily with inflexible restrictions on communication; they allow that, as a last resort, usage-sensitive pricing could be used to discourage electronic junk mail.

As discussed in Section 1, information overload is a problem because senders screen too little and so receivers end up poorly informed. If senders cannot selectively screen (e.g., if the messages are sent by nature), then there is no benefit to reducing the flow of information to receivers. This statement is true, however, only if people can ignore incoming messages and do not systematically err by processing too many messages. Psychologists study the ability of humans to ignore incoming messages, see, for example, Libowski (1975).

In the marketing literature, Jacoby et al. (1974), Malhotra (1982), and Keller and Staelin (1987) have experimentally tested the following hypothesis: consumers choose to process too much information and thus may benefit from unselective restrictions on their access to information. Flaws in this research have been pointed out by critics such as Grether et al. (1986) and Meyer and Johnson (1989). This literature has ignored the inverse relationship between the quantity and quality of information that we study in this paper.

Legal scholars are interested in the practical implications of information overload for disclosure laws, contract complexity, and disclaimers. An extensive discussion, drawing on economic theory, can be found in Schwartz and Wilde (1983).

In our model, the receivers can be viewed as searching among their messages; hence the model is related to the economics literature on consumer search. Our model and results are different from those of that literature, however. Consumer welfare may decline in some search models when there are more firms in the market, but this is because equilibrium prices rise. In Satterthwaite (1979), this occurs because the market is for a differentiated reputation good - the larger the market is, the more costly it is to obtain several referrals about the quality and price of any one product. This makes demand less elastic. In Stahl (1989), the market is for a homogeneous good and consumers search only for price information. A population of consumers with zero search costs keeps prices from the monopolistic price, which would prevail if search were costly for all consumers (Diamond (1971)), but the effect of this population on each firm's price strategy diminishes as the number of firms rises. A similar phenomenon occurs in Rosenthal (1980). In our model, the terms of the opportunities offered by senders are fixed exogenously, but the receivers' welfare may decline when the senders stop screening and when receivers are thus less likely to learn about the opportunities in which they are interested.

A leading interpretation of our model is as advertising (although the price competition is not included in the model). Butters (1977) studies competition between advertisers, but this competition is via prices rather than for the buyers' attention. Identical firms sell a homogeneous good to identical consumers. Each buyer processes all the advertisements she receives and then purchases at the lowest advertised price. In various extensions, Stegeman (1991) makes the consumers heterogeneous, Robert and Stahl (1993) add consumer search as a supplementary information channel, and Stahl (1994) generalizes various assumptions 
on consumer demand and producers' costs. Grossman and Shapiro (1984) study a similar model, also with no limits to consumer attention, in which products and consumer preferences are differentiated. A decrease in advertising costs may reduce profits of the firms, but this is because it heightens price competition rather than because it reduces the effectiveness of communication (as in our model); a reduction in the advertising cost always increases total surplus.

\section{Model}

The first key aspect of communication that we want to capture is the informational difference between a sender of a message, who costlessly knows its contents but is not fully informed about the interests of potential receivers, and a receiver, who costlessly knows his or her own interests but is not fully informed about the contents of messages without first processing them. The second key aspect is the externality between senders of messages as they compete for the scarce attention of receivers. Furthermore, we want to study a network in which communication is targeted rather than broadcast, meaning that messages are sent to individual receivers and that one of the decisions made by senders is whom to target - based on imperfect information about the receiver's interests.

We construct a model that brings these points to the forefront. Communication takes place once and is unidirectional. A finite number $n>1$ of senders, indexed $j=1, \ldots, n$, transmit messages to a large population of receivers. We use male pronouns for senders and female for receivers. Each sender has a single message. It is unstructured, which means that (a) it can be read either in its entirety or not at all and (b) a receiver cannot distinguish between messages when choosing which to read. Each message informs a receiver of an opportunity or recommendation, which we call simply an offer. The terms of the offer are fixed. Hence, a sender's only decision is choosing whom to send messages to. A receiver is not aware of a sender's offer unless she receives and processes his message. A senders' payoff increases as a function of the number of receivers who receive, process, and respond to his message.

Before imposing additional simplifying assumptions, we note the following examples:

1. Senders are organizers of seminars in a university and receivers are faculty and students. Each organizer can send an announcement about the seminar he is organizing to anyone in the university through the intramural mail or by e-mail. The organizer's status depends on the number of attendees.

2. Senders are safety groups in a firm and receivers are employees. Each group can send a recommendation to the employees, using intramural mail or e-mail, about the safety hazards it is responsible for. The groups are evaluated by the safety records.

3. Senders are firms and receivers are consumers. Each firm offers a transaction with exogenously given terms. For example, the transaction might be the sale of a product at an exogenously given price. Each firm can send any set of consumers a description of the transaction, a web address, an order form, or a toll-free number through the mail. The firm's profits depend on how many consumers accept the transaction.

The last example of firms and consumers fits the model well, so we borrow terminology from it.

Senders decide simultaneously whom to target. Each receiver then receives between 0 and $n$ messages. She can process up to $m$ messages costlessly but cannot process more. 
So that information overload is potentially a problem, we assume $1 \leq m<n$. Because messages are indistinguishable when they arrive and cannot be partially processed, each message received is processed with equal probability. In particular, a receiver who receives $l$ messages processes all of them if $l \leq m$ and processes each one with probability $m / l$ otherwise.

This particular search technology is a minor simplification (as compared to sequential search in which a cost is paid for each message processed). More important is our assumption (standard in the screening literature) that messages are unstructured, which precludes screening based on partial processing of messages. Such screening is an important mechanism for dealing with information overload. However, as much as such screening merits study and is important for a measurement of the effects studied in this paper, it does not alter the qualitative points that we make. Each decision by a receiver of whether to process other parts of a message is still made based on partial information about the content and relevance of these parts. For examples, when searching through working papers, being able to read titles in order to decide whether to read abstracts, abstracts in order to decide whether to read introductions, and so on, is a structured search process that improves communication but does not eliminate the possibility that we choose not to read papers that, ex-post, we would have been happy to have read, or that we read papers that, ex-post, we wish we had not bothered with. ${ }^{1}$

After processing up to $m$ messages, the receiver either responds to a message if interested or does not. Whether a receiver is interested in a message does not depend on what other messages she processes. Thus, senders are competing only for the attention of the receiver and not directly with the other offers.

Particularly in the advertising scenario in which senders are firms, the design of the offers (for example, the design and pricing of products) and the direct competitive interaction when the offers are substitutes or complements are interesting issues. We abstract from them because they have been well studied in models without information overload and are not essential to the competition for attention. The interaction between price competition and competition for attention is left for future research.

The most intricate part of our model is our treatment of receivers and of the senders' information about them. We use a reduced form that is motivated informally here and is derived from a primitive model described in Appendix A. Senders cannot perfectly observe which messages a receiver would be interested in. They have common beliefs about this based on, for example, demographic and marketing data. We call these beliefs about a receiver her type and represent them by $t \in[0,1]^{n}$, where $t=\left\langle t_{1}, \ldots, t_{n}\right\rangle$ and $t_{j}$ is the probability that the receiver is interested in sender $j$ 's message. Thus, if sender $j$ sends one of the $l$ messages received by a receiver of type $\left\langle t_{1}, \ldots, t_{n}\right\rangle$, then the probability that the receiver reads $j$ 's message is $\min \{1, m / l\}$ and the probability that she also responds to it is $t_{j}(\min \{1, m / l\})$.

Let $T \equiv[0,1]^{n}$ be the set of types. The distribution of types in the population is given by a measure $\gamma$ on $T$, normalized so that $\gamma(T)=1$. We have in mind a large population, so we typically assume that $\gamma$ is dispersed in some sense. If the demographic characteristics are good indicators of the interests of receivers, then $\gamma$ places greater mass near the corners of $T$, where a type is an $n$-tuple of $0 \mathrm{~s}$ and $1 \mathrm{~s}$ and hence the receiver's interests are known. Otherwise, $\gamma$ is concentrated around its mean. If each receiver is interested in one and

\footnotetext{
${ }^{1}$ The article "There's still no quick fix for dumping the e-mail junk", by Rob Pegararo (International Herald Tribune, 24 July 2000, p. 19), observes that even software filters are imperfect at screening out junk mail: "They cannot catch everything. Worse, yet, sometimes they go too far, trashing the things you do want to see." They are even worse at letting through just the "junk mail" that would be of interest.
} 


\section{Division between basketball and golf players}

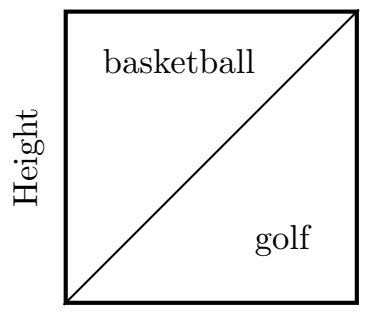

Income

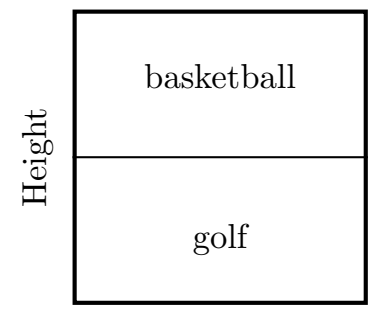

Income

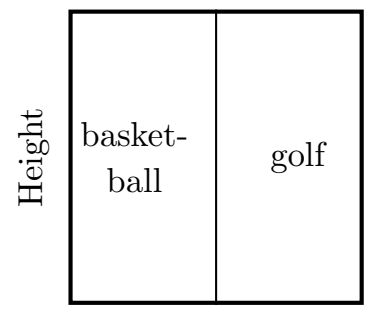

Income

\section{Support of $\gamma$}
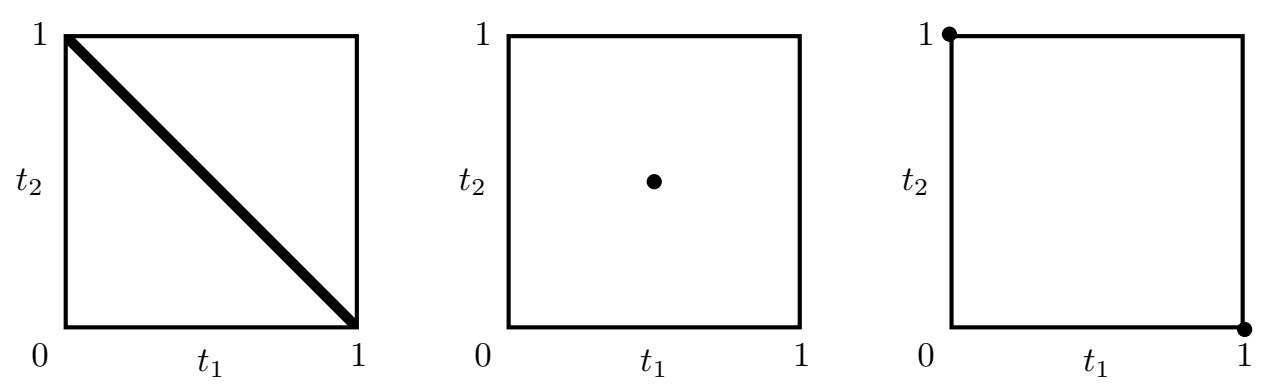

FiguRE 1. Illustration of the senders' information. There are two senders, a basketball equipment retailer and a golf equipment retailer. Each receiver plays either basketball or golf, depending only on her income and height. The receivers' income and heights are distributed uniformly on $[\$ 0, \$ 100 \mathrm{~K}] \times[150 \mathrm{~cm}, 200 \mathrm{~cm}]$. The division between basketball and golf players is shown in the upper row for three cases. The senders know the receivers' incomes but not their heights. The support of the induced distribution $\gamma$ on $[0,1]^{2}$ is drawn in the lower row. For the left-hand division, income is partly informative and $\gamma$ is uniform on the simplex $\Delta^{1}$. For the middle division, income is uninformative and $\gamma$ is concentrated on $\langle 1 / 2,1 / 2\rangle$. For the right-hand division, income is fully informative and $\gamma$ is concentrated on $\langle 1,0\rangle$ and $\langle 0,1\rangle$.

only one message, then the entire mass of $\gamma$ is on the $(n-1)$-dimensional unit simplex $\Delta^{n-1}$. This special case is used for some graphical examples because the set of types has one less dimension. Figure 1 contains an illustration of the link between $\gamma$ and the senders' information; see also Appendix A.

We require that each sender treat receivers of the same type the same way, targeting all or none of them. This is not a strong restriction, given the dispersion of types; senders can divide up a pool of receivers by dividing up similar types rather than dividing up receivers of the same type. Given this restriction, a typical pure strategy for a sender $j$ is a Borel subset $X_{j}$ of $T$, where $X_{j}$ is the set of types that the sender targets. We let $\mathcal{B}$ be the set of Borel subsets of $T$, which thus denotes each sender's set of pure strategies. A strategy profile for the senders is denoted $X=\left\langle X_{1}, \ldots, X_{n}\right\rangle \in \mathcal{B}^{n}$.

The following notation keeps track of how many senders communicate to a given receiver type. Given a strategy profile $X=\left\langle X_{1}, \ldots, X_{n}\right\rangle$, let $X_{-j} \equiv\left\langle X_{1}, \ldots, X_{j-1}, X_{j+1}, \ldots, X_{n}\right\rangle$. For $t \in T$, let $X_{j}(t)=1$ if $t \in X_{j}$ and $X_{j}(t)=0$ otherwise; that is, $X_{j}(\cdot)$ denotes the indicator function of $X_{j}$. Define also $\# X(t) \equiv \sum_{j=1}^{n} X_{j}(t)$ and $\# X_{-j}(t) \equiv \sum_{i \neq j} X_{i}(t)$. 
Hence, $\# X(t)$ is the number of senders who target $t$, and $\# X_{-j}(t)$ is the number of senders other than $j$ who target $t$.

If a receiver processes a message in which she is interested, then she accepts the offer and the sender is said to have made a "sale". The expected sales of sender $j$ given a strategy profile $X$ are

$$
\sigma_{j}(X) \equiv \int_{X_{j}} t_{j}(\min \{1, m / \# X(t)\}) d \gamma(t) .
$$

Invoking informally a law of large numbers and assumptions that the marketing data are unbiased and errors across receivers are i.i.d., we treat expected sales as also realized sales in order to simplify the discussion. We assume that sender $j$ earns a surplus $s_{j}>0$ per transaction, so $j$ 's gross payoff given $X$, before discounting communication costs, is $s_{j} \sigma_{j}(X)$.

Sending messages may be costly for a sender because it requires resources such as envelopes, paper, or bandwidth; these costs are nearly proportional to the number of receivers targeted. The sender also has fixed costs of preparing the original copy of the message and obtaining the mailing lists and marketing data. We disregard these fixed costs. We assume that the cost per message sent is the same for all senders and receivers. Let $c$ be this cost per message, so that the cost of targeting types $B \in \mathcal{B}$ is $c \gamma(B)$. The net payoff of sender $j$ given $X$ and $c$ is then

$$
\pi_{j}(X ; c) \equiv s_{j} \sigma_{j}(X)-c \gamma\left(X_{j}\right) .
$$

Each communication cost $c \geq 0$ thus defines a game $\Gamma^{c}$ in normal form in which the players are the $n$ senders, each sender's strategy set is $\mathcal{B}$, and sender $j$ 's payoff function is $\pi_{j}(\cdot ; c)$. By an equilibrium for $\Gamma^{c}$, we mean a pure-strategy Nash equilibrium. Our task is to examine how the equilibria of $\Gamma^{c}$ depend on $c$.

\section{Equilibrium}

Each sender's surplus is linear in sales, the cost of communication is linear in the number of messages sent, and sales to a receiver depend only on the messages sent to that receiver. Therefore, the game $\Gamma^{c}$ can be decomposed into independent single-receiver games. This is proved in Proposition 4.2 after developing necessary notation and definitions.

For $t \in T$, let $\Gamma^{c}(t)$ be the single-receiver game for type $t$. This is the game in normal form in which: (a) there are $n$ players; (b) each player's strategy set is $\{0,1\}$, where 0 means "not send" and 1 means "send"; and (c) player $j$ 's payoff, given the strategy profile $x \equiv\left\langle x_{1}, \ldots, x_{n}\right\rangle \in\{0,1\}^{n}$, is

$$
u_{j}(x ; c, t) \equiv \begin{cases}s_{j} t_{j}(\min \{1, m / \# x\})-c & \text { if } x_{j}=1, \\ 0 & \text { if } x_{j}=0 .\end{cases}
$$

(Here $\# x$ denotes the number of messages sent, i.e. the cardinality of $\left\{i \mid x_{i}=1\right\}$.) For example, if $n=2$ and $m=1$, then the payoff matrix for $\Gamma^{c}(t)$ is

Sender 2

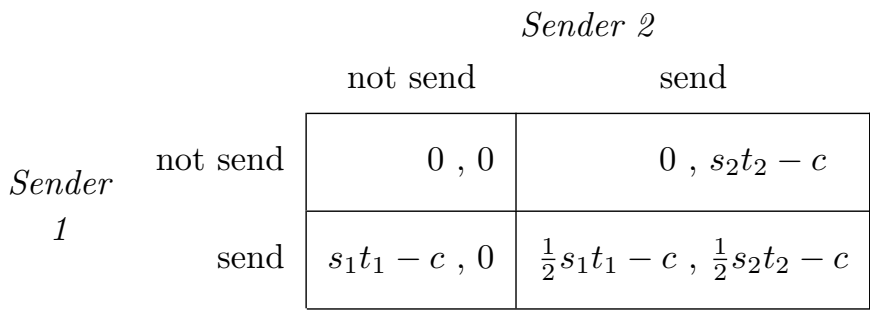




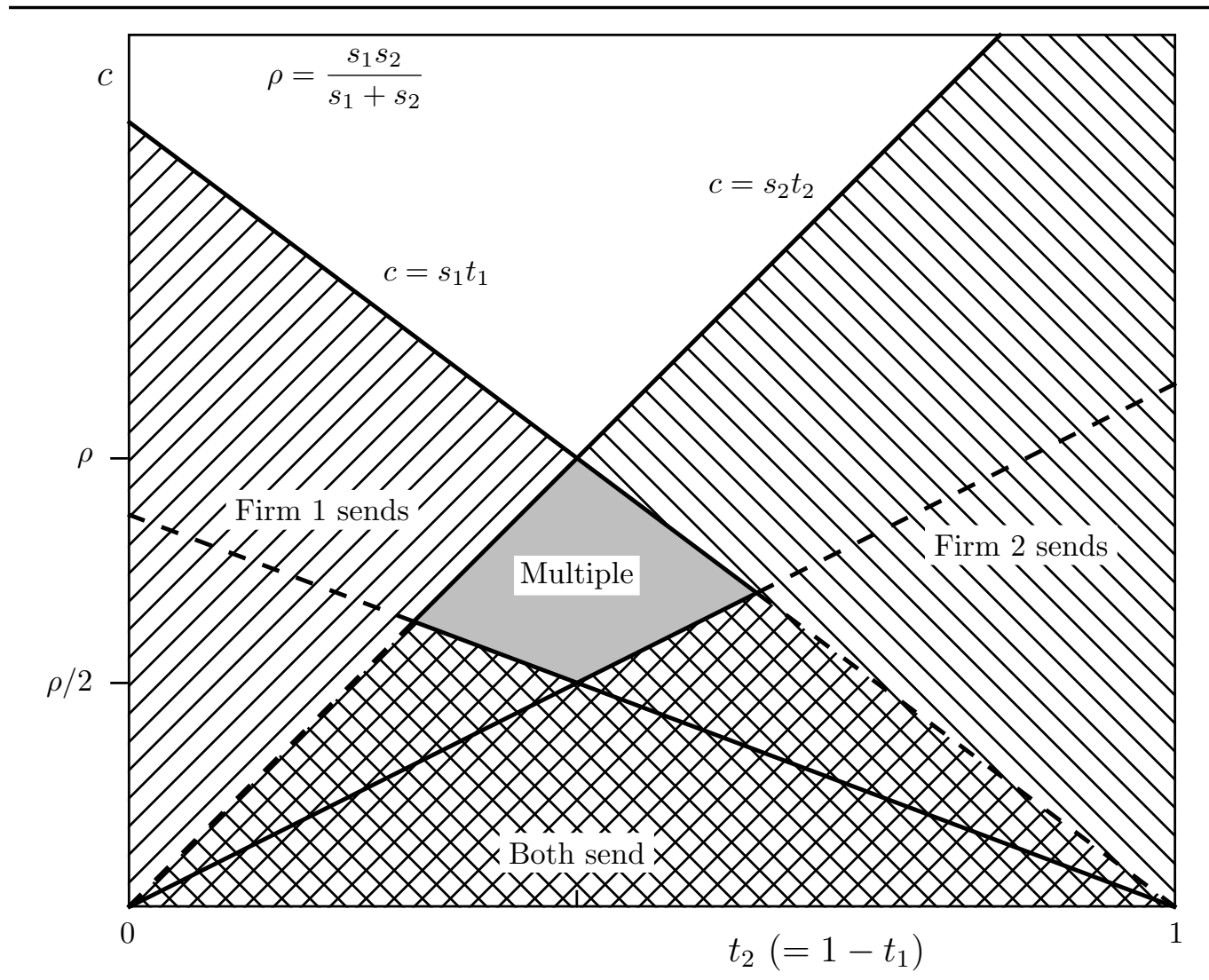

FIgURE 2. Equilibrium as a function of type and communication cost, assuming $n=2$, $m=1, \operatorname{supp}(\gamma)=\Delta^{1}$, and $s_{1}=(3 / 4) s_{2}$. The horizontal axis indicates $t_{2}$ (equal to $\left.1-t_{1}\right)$ and the vertical axis indicates $c$. In the region $\square / \lambda$, only sender 1 sends a message; in the region $\backslash \backslash$, only sender 2 sends a message; in the region $\square \backslash \backslash$, both send a message; and in region $\square$, there are two equilibria in which one and only one of the senders sends a message. In the blank region, there is a unique equilibrium in which no messages are sent.

The game $\Gamma^{c}(t)$ is similar in structure to a costly-entry oligopoly game or to a commons game with only two actions: "exploit" and "not exploit". In particular, each player's payoff from sending a message (exploiting the resource) is decreasing in the number of other players who send a message and is independent of the identities of these players. Consequently, each single-receiver game has a pure-strategy equilibrium, as stated in Proposition 4.1. (All proofs are in Appendix B.)

Proposition 4.1 For all $c \geq 0$ and $t \in T, \Gamma^{c}(t)$ has a pure-strategy equilibrium.

Figure 2 shows the equilibria of $\Gamma^{c}(t)$ as a function of $c$ and $t$ (for particular values of the other parameters).

The aggregate game $\Gamma^{c}$ is similar to an oligopoly game with costly entry and many independent markets or to a commons game with many independent resources to be exploited. A sender's payoff in $\Gamma^{c}$ given a strategy profile $X \equiv\left\langle X_{1}, \ldots, X_{n}\right\rangle$ is equal to the average of its payoffs in the games $\Gamma^{c}(t)$ given $\left\langle X_{1}(t), \ldots, X_{n}(t)\right\rangle$. That is,

$$
\pi_{j}(X ; c)=\int_{T} u_{j}\left(X_{1}(t), \ldots, X_{n}(t) ; c, t\right) d \gamma(t)
$$


Proposition 4.2 then follows easily.

Proposition 4.2 Let $c \geq 0$. A strategy profile $\left\langle X_{1}, \ldots, X_{n}\right\rangle$ is an equilibrium for $\Gamma^{c}$ if and only if, for $\gamma$-a.e. $t \in T,\left\langle X_{1}(t), \ldots, X_{n}(t)\right\rangle$ is a pure-strategy equilibrium for $\Gamma^{c}(t)$.

Corollary 4.1 $\Gamma^{c}$ has an equilibrium for all $c \geq 0$.

To find an equilibrium of $\Gamma^{c}$ for the example in Figure 2, we choose a measurable selection of the correspondence to equilibria of $\Gamma^{c}(t)$.

For points in the boundaries dividing the regions in Figure 2, the set of equilibria of the game $\Gamma^{c}(t)$ consists of the equilibria for the bordering regions. It is convenient to be able to ignore such multiplicities. Assumption 4.1 gives the collection of such points zero mass. It specifically gives zero mass to certain $(n-1)$-dimensional subspaces of $T$, although not to the $(n-1)$-dimensional simplex. This assumption is maintained for the rest of the paper.

Assumption 4.1 For all $i, j \in\{1, \ldots, n\}$ such that $i \neq j$, for all $a \in \mathbb{R}_{+}$, and for all $b \in \mathbb{R}$,

$$
\gamma\left\{t \in T \mid t_{j}=a t_{i}+b\right\}=0 .
$$

Two strategies are said to be equivalent if their symmetric difference is $\gamma$-null. Two strategy profiles are said to be equivalent if, for each sender, the sender's strategies in the two profiles are equivalent. The term "unique" — whether applied to strategies, strategy profiles, or equilibria-means "unique up to equivalence". For example, Assumption 4.1 implies that $\Gamma^{c}$ has a unique equilibrium in the example in Figure 2 if either $c \leq s_{1} s_{2} / 2\left(s_{1}+s_{2}\right)$ or $c \geq s_{1} s_{2} /\left(s_{1}+s_{2}\right)$, because then $\Gamma^{c}(t)$ has a unique equilibrium for $\gamma$-a.e. $t \in T$ (since $\gamma$ has no mass points).

We conclude this section by stating a useful implication of Assumption 4.1: each sender has a unique best response $X_{j}^{*}\left(X_{-j} ; c\right)$ to any strategy profile $X_{j}$ of the other senders.

Proposition 4.3 Let $j \in\{1, \ldots, n\}$ and $c \geq 0$. Let $X_{-j} \equiv\left\langle X_{1}, \ldots, X_{j-1}, X_{j+1}, \ldots, X_{n}\right\rangle \in$ $\mathcal{B}^{n-1}$ be a profile of strategies for senders other than $j$. Then sender $j$ has a unique best response to $X_{-j}$ given $c$. That is, $\max _{X_{j} \in \mathcal{B}} \pi_{j}\left(X_{j}, X_{-j} ; c\right)$ has a solution and it is unique up to equivalence. Denote this solution by $X_{j}^{*}\left(X_{-j} ; c\right)$.

\section{$5 \quad$ Strategies that maximize the senders' total payoffs}

We can evaluate the outcomes of this communication game from the point of view of the senders, of the receivers, or of both. When receivers are overloaded with information, they feel victimized by the senders - ask anyone how they feel about the companies that send junk postal and electronic mail. However, senders are also hurt by information overload because the receivers' attention is not going to those senders who value it the most. In Sections 5-7, we focus on the senders.

The following notation is useful. For $t \in T$ and $j \in\{1, \ldots, n\}$, define sender $j$ 's valuation of $t$ 's attention to be $s_{j} t_{j}$ and denote it by $v_{j}(t)$. It equals $j$ 's expected gross payoff in the game $\Gamma^{c}(t)$ conditional on the receiver's processing his message. For $l \in\{1, \ldots, n\}$, let $v^{l}(t)$ be the $l$ th highest valuation of $t$ 's attention; this is, if the senders are numbered such that $v_{1}(t) \geq \cdots \geq v_{n}(t)$, then $v^{l}(t)=v_{l}(t)$.

We observe that there is a unique strategy profile $Y^{c}$ that maximizes the total net payoffs of the senders in the game $\Gamma^{c}$. We call $Y^{c}$ the efficient strategy profile, keeping in mind that 


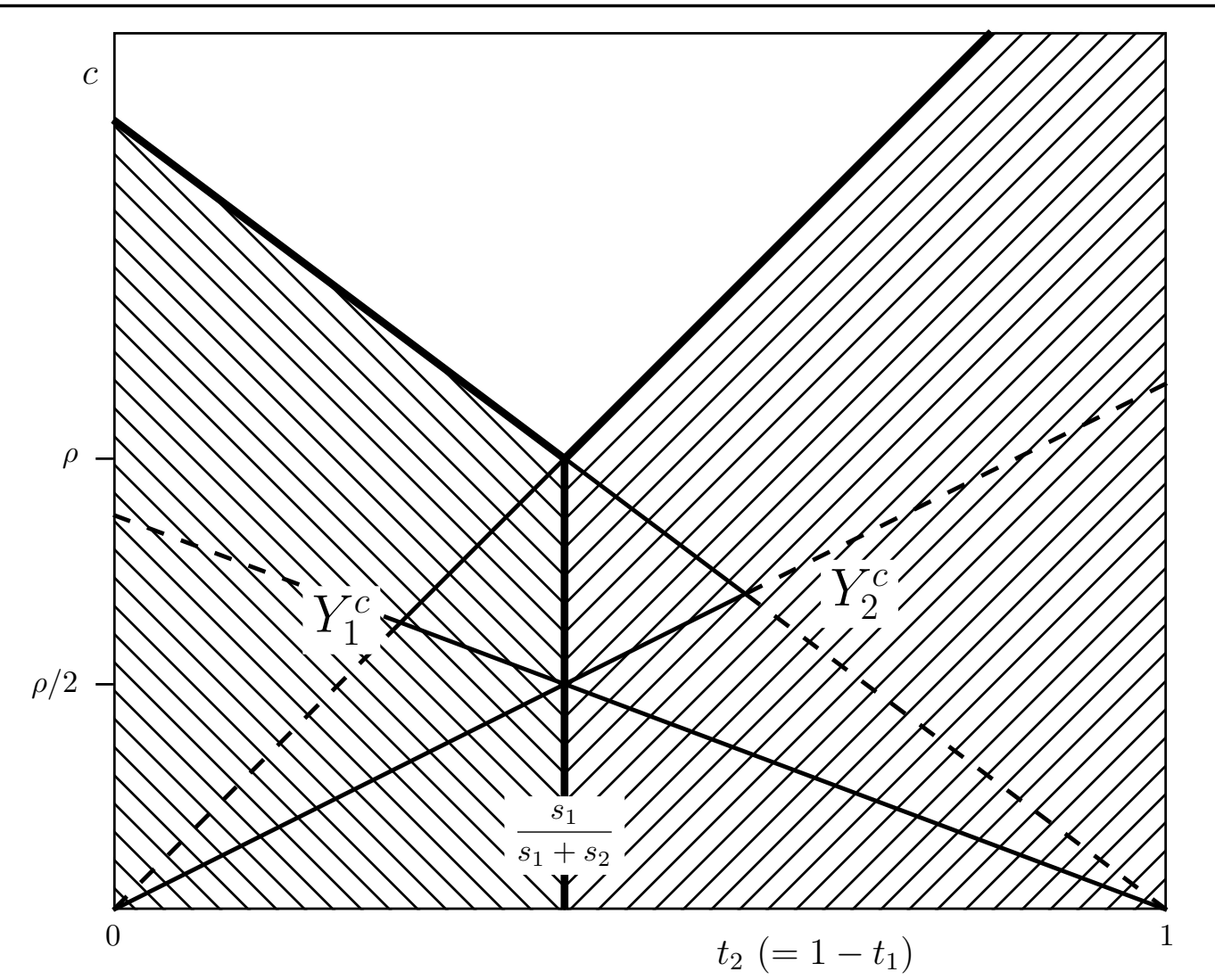

FIgURE 3. Efficient communication (from point of view of senders) as a function of type and communication cost, assuming $n=2, m=1, \operatorname{supp}(\gamma)=\Delta^{1}, s_{1}=(3 / 4) s_{2}$. Sender 1 sends a message in the left-hand region $Y_{1}^{c}$ delineated by the heavy line, and sender 2 sends a message in the right-hand region $Y_{2}^{c}$.

efficiency is with respect to only the senders' payoffs. The profile $Y^{c}$ is defined as follows. It has no information overload; instead, for $\gamma$-a.e. $t \in T$, type $t$ is targeted by the $m$ senders with the highest valuations, except those whose valuations do not exceed $c$. This condition defines a unique strategy profile of the aggregate game because the $n$ senders have distinct valuations for $\gamma$-a.e. $t \in T$ (due to Assumption 4.1). Sender $j$ 's strategy in this profile is

$$
Y_{j}^{c} \equiv\left\{t \in T \mid v_{j}(t) \geq \max \left\{c, v^{m}(t)\right\}\right\} .
$$

The boundaries of $Y^{c}$ are drawn in Figure 3 for the example illustrated in Figure 2, in which $\gamma$ is concentrated on $\Delta^{1}$. When the communication cost $c$ is less than or equal to $\rho=$ $s_{1} s_{2} /\left(s_{1}+s_{2}\right)$, sender 2 optimally targets those receivers lying to the right of $s_{1} /\left(s_{1}+s_{2}\right)$ in the figure and sender 1 optimally targets those lying to the left of $s_{1} /\left(s_{1}+s_{2}\right)$. Compare this with the equilibrium shown in Figure 2. When the communication cost is zero, each sender communicates to all receivers (the dominant strategy) and all receivers are overloaded. As the communication cost increases from zero, the set of receivers who receive more messages than they can process decreases as each sender stops communicating to receivers who are unlikely to be interested in his message; however, there is still more communication than in $Y^{c}$ for any communication cost less than $\rho / 2$. When the communication cost reaches $\rho / 2$, the two senders partition the set of receivers efficiently in equilibrium. When the communication cost is between $\rho / 2$ and $\rho$, there are multiple equilibria. In any equilibrium, the two senders still partition the set of receivers and hence receivers are not overloaded. 
Furthermore, $Y^{c}$ is one of the equilibria. However, there exist other inefficient equilibria. When the communication cost is greater than $\rho, Y^{c}$ is the unique equilibrium.

The two main ideas (familiar from commons games) that this example illustrates are: (a) there may be too much (but not too little) communication in equilibrium because the senders' messages have a negative externality on each other - each sender's message crowds the other messages; and (b) the communication cost is a natural rationing mechanism, so excessive communication arises in equilibrium only when the communication cost is too low. We summarize these ideas formally in Proposition 5.1. Part 1 says that the total communication in equilibrium is greater than or equal to the total communication given $Y^{c}$. Part 2 says that if senders other than $j$ adopt the strategies $Y_{-j}^{c}$, then sender $j$ wants to target at least the receivers in $Y_{j}^{c}$ and perhaps others. Part 3 says the strategy profile that maximizes the senders' profits is an equilibrium if and only if the communication cost is high enough.

\section{Proposition 5.1}

(1) Let $c \geq 0$ and let $\left\langle X_{1}, \ldots, X_{n}\right\rangle$ be an equilibrium for $\Gamma^{c}$. Then $\sum_{j=1}^{n} \gamma X_{j} \geq \sum_{j=1}^{n} \gamma Y_{j}^{c}$.

(2) Let $c \geq 0$ and let $j \in\{1, \ldots, n\}$. Then $Y_{j}^{c} \subset X_{j}^{*}\left(Y_{-j}^{c} ; c\right)$.

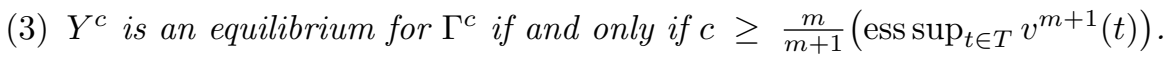

\section{Type-dependent mechanisms for allocating attention}

If we can allocate attention using a mechanism that treats each receiver type independently, then we have a family of independent mechanism design problems. Standard mechanisms for solving a tragedy of the commons can then be used to support $Y^{c}$.

For example, suppose the mechanism designer has the same information as the senders. Then the designer can use a price mechanism $P: T \rightarrow \mathbb{R}_{+}$so that the surcharge on targeting $B \subset T$ is $\int_{B} P(t) d \gamma$. This defines a game $\Gamma^{(c, P)}$ in which each sender's strategy set is $\mathcal{B}$ and sender $j$ 's payoff, given $X \in \mathcal{B}^{n}$, is $s_{j} \sigma_{j}(X)-\int_{X_{j}}(c+P(t)) d \gamma$.

To make $Y^{c}(t)$ an equilibrium in the game $\Gamma^{c}(t)$, we need to add a surcharge that is just large enough to deter the sender with the $(m+1)$ th highest valuation of $t$ 's attention from sending a message, given that $m$ other senders also send messages. This condition holds when $v^{m+1}(t)-c-P(t) \leq 0$, so we define $P(t)=\max \left\{0, v^{m+1}(t)-c\right\}$. Then $Y^{c}$ is an equilibrium for $\Gamma^{(c, P)}$.

There are two problems with such a mechanism. First, in order to determine $P$, the post office or network manager must know the descriptions of the senders and the marketing data. Second, it is burdensome to implement: to bill the senders it must keep track not only of the bulk quantity of each sender's messages but also of the identities or demographic characteristics of the receivers. With this information and accounting, the network could support $Y^{c}$ simply by deciding which messages get delivered and to whom.

If we ignore the complexity of the mechanism and consider only the possibility that the senders' parameters and marketing data are their private information, then we can use a simple bidding mechanism to allocate the $m$ channels of each receiver. (Using bids to allocate attention was suggested, for example, by Denning (1982).) For a typical receiver, this is a multiunit private-value auction in which each sender wants at most one unit of the attention. The cost of the transmission channels must be zero so that every sender will submit a bid for each receiver; hence, assume in the rest of this section that $c=0$. 
We consider a uniform-price auction as in Vickrey (1961). Each sender writes a bid on each message it sends. For each receiver, the network delivers the messages addressed to her that have the $m$ highest strictly positive bids, choosing randomly in case of a tie. It charges to each sender whose message is delivered the value of the $(m+1)$ th highest bid, or 0 if no more than $m$ messages are sent to the receiver. Then each sender's strategy set is the set of measurable bidding functions from $T$ into $\mathbb{R}_{+}$. It is a dominant strategy for player $j$ to bid his true valuation $v_{j}(t)$. In this dominant-strategy equilibrium, player $j$ 's messages are delivered to receivers in $Y_{j}^{0}$ and the amount paid by the winners for the attention of type $t$ is $v^{m+1}(t)$. Recall that when $c=0, v^{m+1}(\cdot)$ is the receiver-dependent price defined earlier in this section for supporting $Y^{c}$.

Presumably receivers could also process the bids at some cost. If the screening in this bidding game were decentralized in this way, then the outcome would be different because receivers could use their information about their own interest when screening. A discussion of screening by receivers and using bidding or other mechanisms to induce truthful structuring of messages is left for other research. Let us simply note that senders do attach implicit bids to their messages as signals about the importance of their messages. For example, an advertiser might offer a free sample or a sweepstakes if the receiver reads an advertisement.

\section{Allocating attention through uniform surcharges}

Both the type-dependent price mechanism and the bidding mechanism are complex to design and execute. Therefore, we consider what can be achieved using a surcharge on communication that is the same for all types of receivers. It is no longer possible to decompose the model into independent games for the different types. We say that a surcharge $p \geq 0$ supports $Y^{c}$ if $Y^{c}$ is an equilibrium of the game $\Gamma^{(c+p)}$.

Propositions 7.1 and 7.2 show that often, but not always, there is no surcharge that supports $Y^{c}$ when it is not an equilibrium for $\Gamma^{c}$. The negative result in Proposition 7.1 is because $Y_{j}^{c}$ contains receivers who are "marginal" for a sender $j$. That is, for arbitrarily small $\epsilon>0$, there is $t \in Y_{j}^{c}$ such that $s_{j} t_{j}-c<\epsilon$. Any strictly positive surcharge on communication for sender $j$ causes that sender to stop targeting some of these receivers. Thus, a surcharge cannot be used to support $Y^{c}$. This is illustrated in Figure 4.

(As usual, by the support of $\gamma$ we mean the smallest closed set $B \subset T$ such that $\gamma(B)=1$; we denote it by $\operatorname{supp}(\gamma)$. It is the intersection of all closed sets that have measure 1 and it also has measure 1. Furthermore, any non-empty and relatively open subset of $\operatorname{supp}(\gamma)$ has strictly positive measure.)

Proposition 7.1 Let $c \geq 0$. Assume that $Y^{c}$ is not an equilibrium for $\Gamma^{c}$. There is no surcharge that supports $Y^{c}$ if either

(1) $\operatorname{supp}(\gamma)=T$, or

(2) $\Delta^{n-1} \subset \operatorname{supp}(\gamma)$ and either $m>1$ or $s_{j} \leq c$ for some $j$.

When $\operatorname{supp}(\gamma)=\Delta^{n-1}$ and $m=1$, there may not be any marginal receivers; so, if small enough, a surcharge does not induce senders to drop receivers whom they should target in the efficient profile. The difficulty is that, in order to support $Y^{c}$, a surcharge must be large enough to eliminate information overload. Proposition 7.2 delineates the parameter values for which these two requirements can be reconciled. 


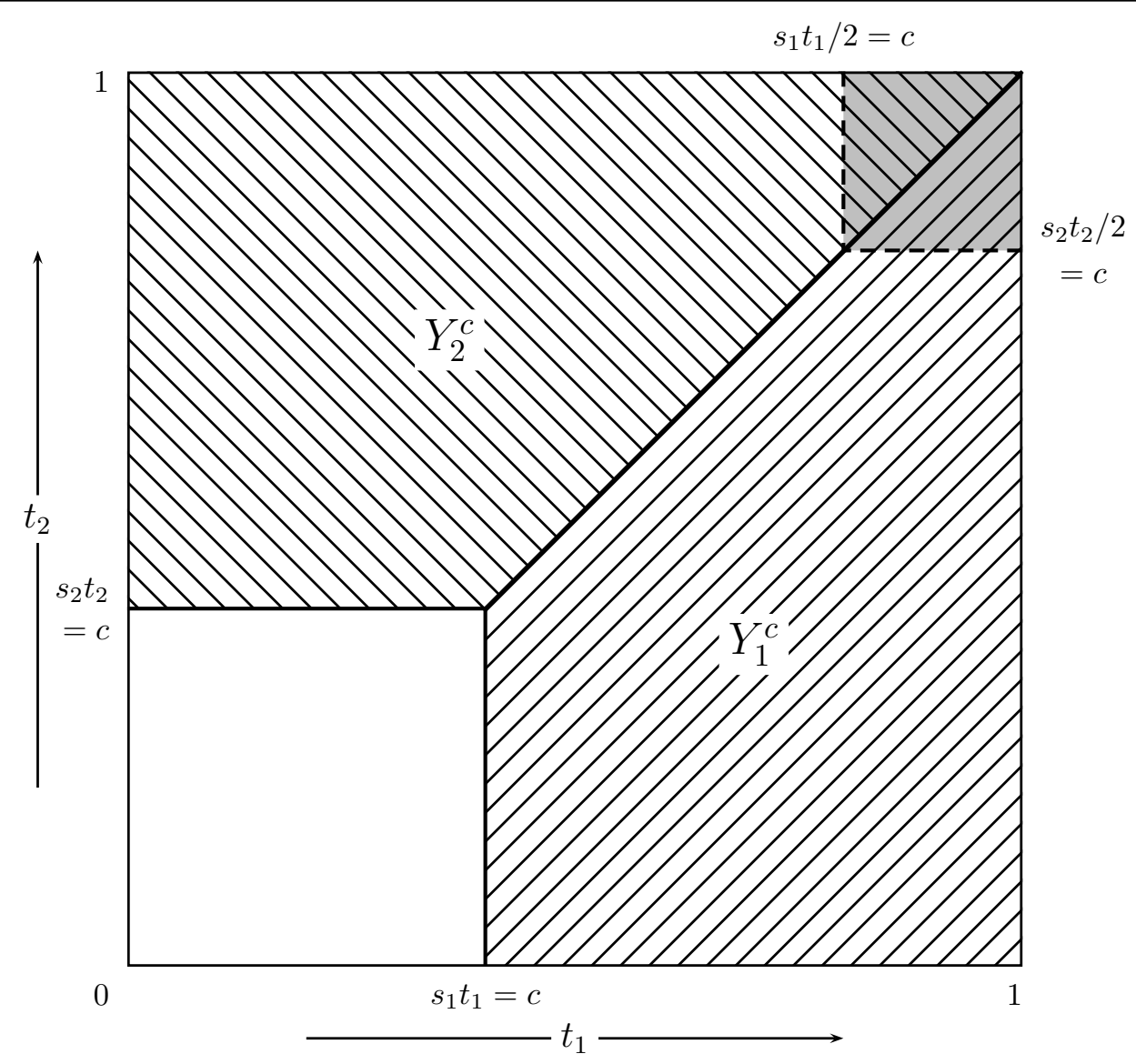

FIgURE 4. Efficient versus equilibrium communication as a function of type, for $n=2$, $m=1, \operatorname{supp}(\gamma)=[0,1]^{2}, s_{1}=s_{2}=1$, and $c=2 / 5$. In the efficient strategy profile, sender 1 sends to types in the region $\square \backslash \square$ and sender 2 sends to types in the region $\triangle \backslash$. In equilibrium, both senders also send a message to types in the region

This overloading can be eliminated by a surcharge, but then the senders will no longer target receivers lying close to the lower left-hand blank square. 
Proposition 7.2 Assume $\operatorname{supp}(\gamma)=\Delta^{n-1}$ and $m=1$. Let $c \geq 0$ and suppose that $Y^{c}$ is not an equilibrium for $\Gamma^{c}$. Then there is a surcharge that supports $Y^{c}$ if and only if either

(1) $n=2$;

(2) $n=3$ and $s_{i}^{-1}+s_{j}^{-1} \geq s_{k}^{-1}$ for all distinct $i, j, k$; or

(3) $n=4$ and $s_{1}=s_{2}=s_{3}=s_{4}$.

In this case, the surcharge $\left(\sum_{k=1}^{n} s_{k}^{-1}\right)^{-1}-c$ supports $Y^{c}$.

\section{Sales and payoffs when the communication cost is low}

This section performs a comparative statics exercise: How do the senders' sales and net payoffs depend on the cost of communication in a neighborhood of zero? Our answer is summarized in Proposition 8.1, the most important result in this paper.

This exercise has two interpretations. In the first, we consider the cost of communication to be a resource cost rather than an artificially imposed surcharge. We ask whether senders benefit from a fall in the cost of communication when this cost is already low. The answer is that the fall in the cost of communication actually reduces all senders' sales; furthermore, if the senders' marketing data are sufficiently accurate, it can even reduce all senders' net profits even though they save on communication costs. This highlights the role that the communication cost plays in rationing receivers' attention.

In the second interpretation, we interpret the cost of communication to be a surcharge, presuming that the resource cost of communication is negligible. We ask whether senders benefit from an increase in the surcharge. The answer is that an increase in the surcharge increases all senders' sales and hence all senders can benefit if the tax is redistributed as lump-sum payments; furthermore, if the senders' marketing data are good enough, all senders' net payoffs can increase even if the surcharge is not returned to them. Thus, although the message of the preceding section is that uniform surcharges on communication cannot generally support efficient communication strategies, we show in this section that the optimal uniform surcharge is strictly positive if the resource cost of communication is low enough.

To quantitatively aggregate sales and payoffs over receiver types, we assume the existence of certain marginal densities for $\gamma$ (but we do not simply assume that $\gamma$ has a density, because this would preclude $\left.\operatorname{supp}(\gamma)=\Delta^{n-1}\right)$.

\section{Assumption 8.1}

(1) For all $j \in\{1, \ldots, n\}$, the marginal distribution of $t_{j}$ has a continuous density $f_{j}$ such that $f_{j}(0)>0$.

(2) For all $i, j \in\{1, \ldots, n\}$ such that $i \neq j$, there is a continuous version of $E\left[t_{i} \mid t_{j}\right]$ such that $E\left[t_{i} \mid t_{j}=0\right]>0$.

(3) If $n>2$, then there is some $k$ such that, for all $i, j \in\{1, \ldots, n\}$ such that $i \neq j$ and for all $\bar{t} \geq 0, \gamma\left\{t \in T \mid t_{i} \leq \bar{t}\right.$ and $\left.t_{j} \leq \bar{t}\right\} \leq k \bar{t}^{2}$.

Proposition 8.1 shows that each sender's equilibrium sales are strictly increasing in the cost of communication in a neighborhood of zero and that, if senders have accurate enough information about the receivers, then payoffs are also strictly increasing in a neighborhood of zero. Because there may be multiple equilibria, we must characterize lower and upper bounds on payoffs. A pictorial version is given in Figure 5 . 


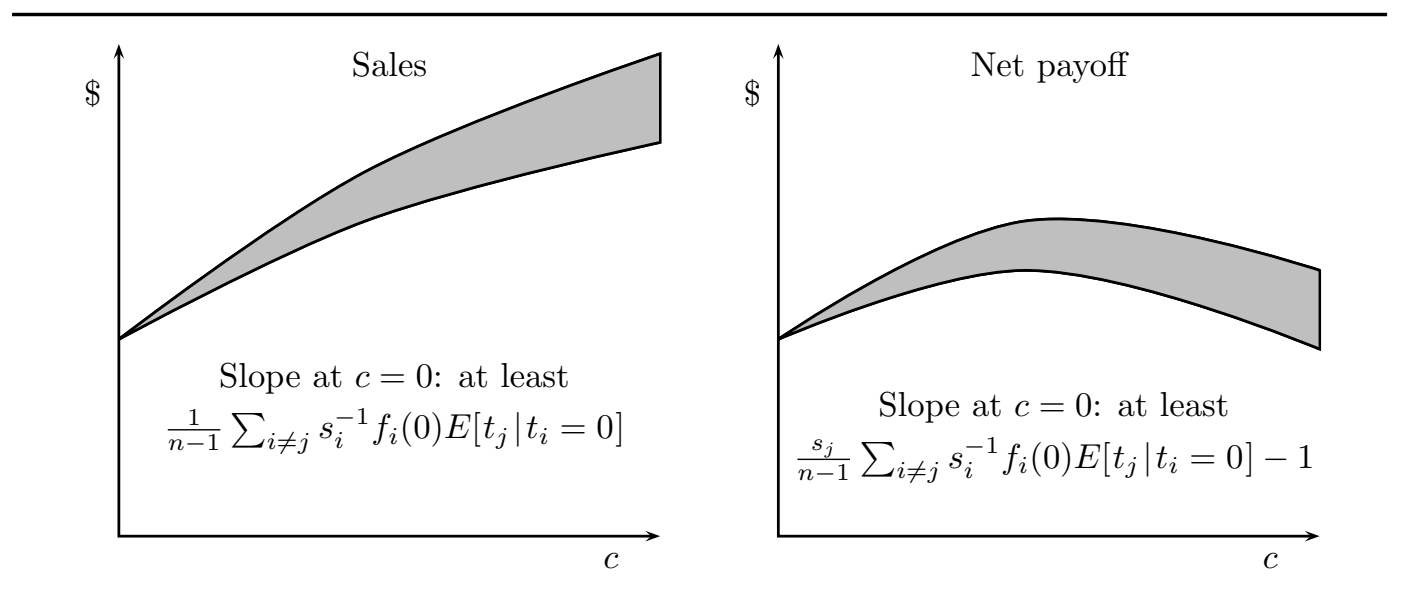

Figure 5. Total sales and net payoffs for a sender as a function of the communication cost.

Proposition 8.1 Let $j \in\{1, \ldots, n\}$. For $c \geq 0$, let $\Sigma_{j}^{+}(c)$ be the maximum sales, let $\Sigma_{j}^{-}(c)$ be the minimum sales, let $\Pi_{j}^{+}(c)$ be the maximum net payoff, and let $\Pi_{j}^{-}(c)$ be the minimum net payoff for sender $j$ in any equilibrium of $\Gamma^{c}$. Then the following statements hold.

(1) $\Sigma_{j}^{+}, \Sigma_{j}^{-}, \Pi_{j}^{+}$, and $\Pi_{j}^{-}$are well-defined continuous functions.

(2) $\Sigma_{j}^{+}(0)=\Sigma_{j}^{-}(0)$ and $\Pi_{j}^{+}(0)=\Pi_{j}^{-}(0)$.

(3) There is a continuously differentiable lower bound $B_{j}: \mathbb{R}_{+} \rightarrow \mathbb{R}_{+}$on $\Sigma_{j}^{-}$such that $B_{j}(0)=\Sigma_{j}^{-}(0)$ and

$$
B_{j}^{\prime}(0)=\frac{1}{n-1} \sum_{i \neq j} \frac{1}{s_{i}} f_{i}(0) E\left[t_{j} \mid t_{i}=0\right]>0 .
$$

(4) There is a continuously differentiable lower bound $\hat{B}_{j}: \mathbb{R}_{+} \rightarrow \mathbb{R}_{+}$on $\Pi_{j}^{-}$such that $\hat{B}_{j}(0)=\Pi_{j}^{-}(0)$ and

$$
\hat{B}_{j}^{\prime}(0)=s_{j} \frac{1}{n-1} \sum_{i \neq j} \frac{1}{s_{i}} f_{i}(0) E\left[t_{j} \mid t_{i}=0\right]-1
$$

Hence, if

$$
\frac{1}{n-1} \sum_{i \neq j} \frac{1}{s_{i}} f_{i}(0) E\left[t_{j} \mid t_{i}=0\right]>\frac{1}{s_{j}},
$$

then $\hat{B}_{j}^{\prime}(0)>0$.

Proposition 8.1 is illustrated in Figure 6 for $n=2, m=1$, and $s_{1}=s_{2}$. The equilibria are drawn for a small communication $\operatorname{cost} c>0$. Each regions is labeled according to which senders target the receivers in that region. If instead $c=0$ then both senders target all receivers. When the cost of communication increases from 0 to $c$, sender 1 loses some sales because he stops targeting receivers in the region labeled " 2 ". However, this loss in sales is negligible because these receivers are unlikely to be interested in his message anyway. On the other hand, sender 1's sales to receivers in the region labeled "1" increase because sender 2 stops targeting these receivers. As long as $E\left[t_{1} \mid t_{2} \leq 2 c / s_{2}\right]>0$, many of these receivers 


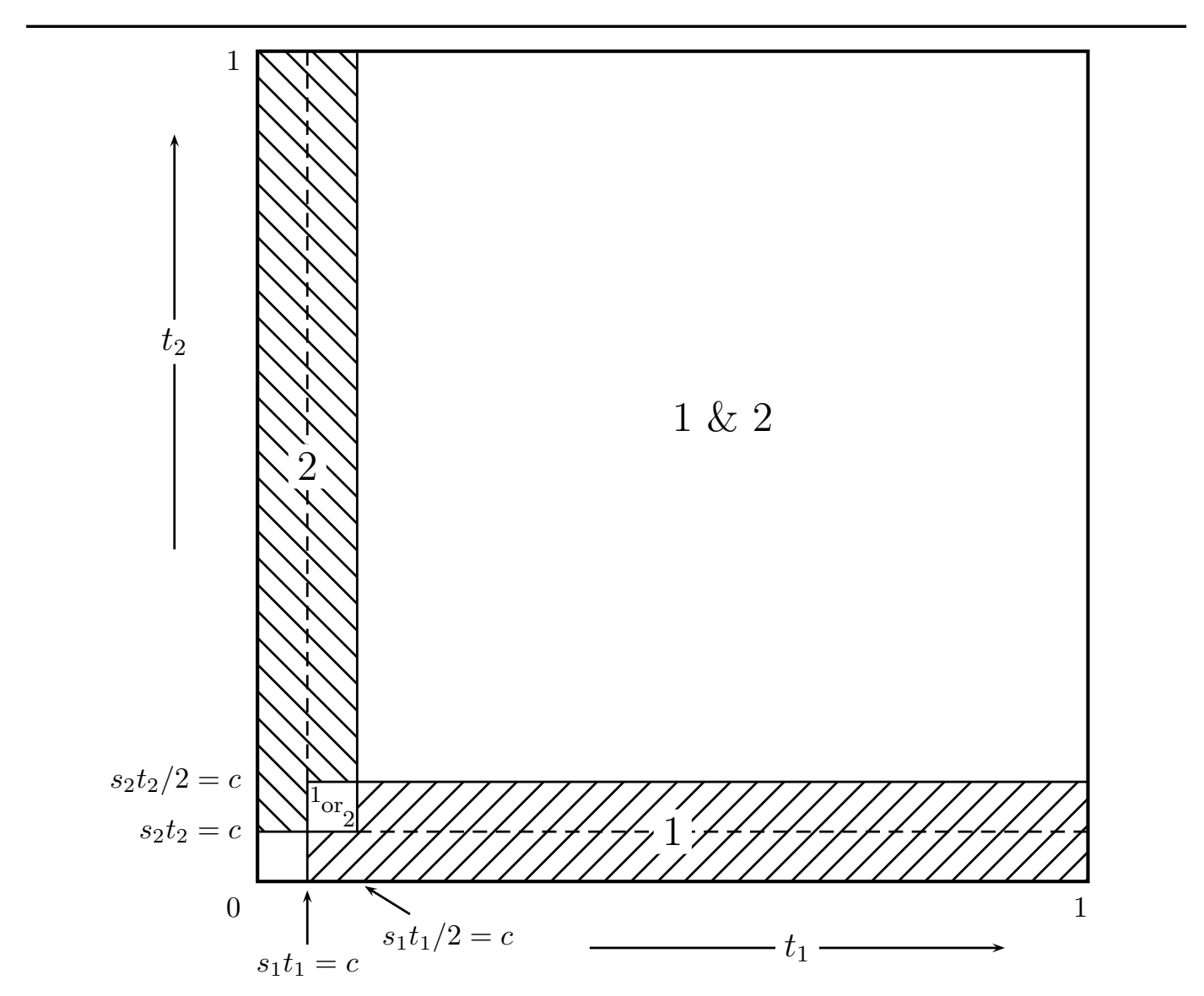

Figure 6. An illustration of why an increase in communication costs from 0 raises sales, for $n=2, m=1$, and $s_{1}=s_{2}$. See the explanation following Proposition 8.1. 
are likely to be interested in sender 1's message, and the mass of receivers in this region is strictly positive if $f_{2}(0)>0$. In this case, the increase in sales is not negligible and is greater than the previously mentioned decrease in sales. If the net increase in sales produces an increase in revenues that is greater than the increase in the cost of communication, then the sender's payoff also increases.

The more accurate the marketing data, the more mass is concentrated near the vertices of $T$ and thus the more the senders' sales and payoffs increase when information overload is reduced. On the other hand, if the mass is concentrated near the mean of $\gamma$, then there is little benefit to reducing information overload. Although senders know the contents of their messages, they are good screeners only to the extent that they have information about the receivers.

\section{Receiver welfare and the cost of communication}

We have not modeled the receivers' behavior explicitly, but we can at least say that a receiver benefits from a recommendation or offer if and only if she accepts it. This circumstance is also precisely when the sender benefits.

Increasing the cost of communication never leads to a Pareto improvement for receivers. When a sender restricts his communication, he stops targeting those receivers who, according to the marketing data, are not likely to be interested in his message. However, because the marketing data are not perfect, some of these receivers would be interested in the message; they end up worse off because they do not receive it.

For example, suppose that there is one record club for rock music and another for classical music. If direct-mail advertising is not free, then the classical club may not target 14-yearold males because most are interested only in rock music. Yet some of these receivers prefer classical to rock; they would be better off choosing randomly between the mailings for rock and classical music rather than only receiving mail from the rock club.

However, the mass of such receivers is small. Most receivers who are no longer targeted by the classical club are interested in rock music, and they benefit from being sure that the mail they process is from the rock club. This difference in numbers is more pronounced the better is the senders' information about receivers.

Suppose that aggregate receiver welfare is measured in the following way. For each recommendation or offer $j$, there is a receiver surplus $\omega_{j}$ (measured in dollars or utils) that represents the benefit to a receiver who adopts the recommendation or offer. Receiver welfare, given the strategy profile $X$, is the total receiver surplus and is equal to

$$
W(X) \equiv \sum_{j=1}^{n} \omega_{j} \sigma_{j}(X) .
$$

If the weights $\omega_{1}, \ldots, \omega_{n}$ are proportional to the per-unit payoffs $s_{1}, \ldots, s_{n}$, then receiver welfare is proportional to the total gross payoffs of the senders. We then say that the interests of the senders and of the receiver coincide. If the cost of transmission channels is zero, then the gross payoffs equal the net payoffs and the bidding mechanism discussed in Section 6 supports the strategies that maximize receiver welfare.

Even if the interests of senders and of receivers diverge, receiver welfare can be increased by raising the cost of communication and thereby reducing information overload. According to Proposition 8.1, if the cost of communication is initially low and if, for each sender $j$, there is a distinct sender $i$ such that $f_{i}(0)>0$ and $E\left[t_{j} \mid t_{i}=0\right]>0$, then there is an increase 
in the cost of communication that leads to an increase in the sales of each sender and thus to an increase in receiver welfare.

On the other hand, if the cost of communication is high, senders may communicate too little because they alone bear the cost of communication and they do not take receiver welfare into account. Suppose that the welfare of receivers and the payoffs of senders are comparable and that we define total welfare (given $X$ ) to be

$$
W^{\prime}(X) \equiv \sum_{j=1}^{n}\left(s_{j}+\omega_{j}\right) \sigma_{j}(X)-c \gamma\left(X_{j}\right) \text {. }
$$

Suppose also that the cost of communication is so high that, although some receivers receive mail, no receivers are overloaded and some receivers receive fewer than $m$ messages. Then the strategy profile that maximizes the senders' total net payoffs is an equilibrium, but total welfare can be increased by expanding the scope of communication. This would be a reason to subsidize communication or to shift the cost of communication from senders to receivers when the cost of communication is high.

\section{Remarks}

The cost of transmission channels serves to allocate receiver input channels in communication networks. As these costs fall, the receiver input channels become relatively more scarce and receivers are overloaded with information. We showed in Sections 8 and 9 that, in the absence of mechanisms for allocating the attention of receivers, all senders and many receivers in a network of targeted communication may become worse off when the cost of transmission channels falls.

This should not discourage efforts to reduce the cost of such channels; rather, it should encourage the design and adoption of mechanisms for allocating attention. We have studied how price and bidding mechanisms can increase the efficiency of communication. Our results suggest, for example, that one way to reduce the burden of sorting through junk mail is to increase the postal rate for bulk mail. Advertisers will then target consumers more selectively, which may both increase their sales and benefit the consumers.

Of course, this paper models only a specific class of networks under fairly severe restrictions. For example, in our model, the messages were homogeneous. In practice, a mail network handles personal letters, solicited bulk mail, unsolicited commercial bulk mail, and unsolicited non-profit bulk mail. Since any correspondence can pass for a personal letter, the price of bulk mail cannot exceed the first-class rate for personal letters. There is no reason to increase the cost of sending a personal letter, since the sender has already incurred a large cost in writing it. There is no reason to increase the cost of solicited communication, because the receiver has already made an informed decision to allocate his attention to the message. The inability of the network to differentiate perfectly among these types of mail constrains the feasibility of mechanisms.

Messages were not structured in our model. The allocation of attention when messages are structured is particularly interesting when senders can lie or use deceptive structuring, such as writing "Regional Weather Alert" on an advertisement for roofing or having the words "pay to the order of" appear in the address window. We need mechanisms that induce truthful, structured messages. The bidding mechanism discussed in Section 6 is an example. 


\section{A Demographic data and types}

This appendix describes a more primitive model of the receivers and the information the senders have about them. Because the purpose is to provide intuition rather than a mathematical framework to be used elsewhere in the paper, we consider a finite model. The model with a large dispersed population of receivers and types of receivers is meant to be an approximation of this finite model.

There is a finite set $A$ of receivers. The senders have a common mailing list, which gives the name and address of each receiver. The mailing list also gives demographic information such as age, sex, race, place of residence, job title, and magazine subscriptions. Let $D$ be the finite set of possible demographic characteristics. Let $Z: A \rightarrow D$ be a function that specifies the characteristic of each receiver.

The senders also have marketing data, which gives the correlation between these characteristics and the interest in the senders' messages. Specifically, let $A_{j} \subset A$ be the set of receivers who are interested in $j$ 's message. For $d \in D$, let

$$
\delta_{j}(d) \equiv \frac{\#\left(A_{j} \cap Z^{-1}(d)\right)}{\# Z^{-1}(d)} .
$$

Here, $\delta_{j}(d)$ is the proportion of those receivers with demographic characteristic $d$ who are interested in $j$ 's message. Sender $j$ can estimate $\delta_{j}(d)$ by sampling the population of receivers in $Z^{-1}(d)$, such as with marketing surveys.

Let $\delta(d)=\left\langle\delta_{1}(d), \ldots, \delta_{n}(d)\right\rangle$ and let $T \equiv[0,1]^{n}$ be the set of types. Then $\delta: D \rightarrow T$. Let $M: A \rightarrow T$ be the composition $\delta \circ Z$. Receiver a's type is $M(a)$. The number of receivers of type $t \in T$ is $\gamma(t) \equiv \# M^{-1}(t)$. The proportion of receivers of type $\left\langle t_{1}, \ldots, t_{n}\right\rangle$ interested is sender $j$ 's message is exactly $t_{j}$.

If the demographic characteristics are good indicators of the interests of the receivers, then $\gamma$ places greater mass near the corners of $T$. Otherwise, $\gamma$ places greater mass near $\left\langle \# A_{1} / \# A, \ldots, \# A_{n} / \# A\right\rangle$. If each receiver is interested in one and only one message, then $\gamma$ puts positive weight only on points in $\Delta^{n-1}$.

\section{B Proofs}

Proof of Proposition 4.1. We use the following structure of the game: (a) each player has a fixed payoff of 0 from not sending a message and (b) each player's payoff from sending a message is decreasing in the number of other players who also send a message but does not depend on the identities of those players.

Let $c \geq 0$ and $t \in T$. For $j \in\{1, \ldots, n\}$, let $l_{j} \in\{0,1, \ldots, n\}$ be such that sending a message is a best response for player $j$ in $\Gamma^{c}(t)$ if and only if at most $l_{j}-1$ other players send messages. Specifically,

$$
l_{j} \equiv \max \left\{l=0,1, \ldots, n \mid l=0 \text { or } s_{j} t_{j}(\min \{1, m / l\})-c \geq 0\right\} .
$$

Renumber the players if necessary so that $l_{1} \geq \cdots \geq l_{n}$. Let $k$ be the highest numbered player $j$ for whom it is optimal to send a message given that players $1, \ldots, j-1$ also send a message. Specifically,

$$
k \equiv \max \left\{j=0,1, \ldots, n \mid j=0 \text { or } l_{j} \geq j\right\}
$$

Let $x$ be the strategy profile such that players $1, \ldots, k$ send messages and the remaining players do not. That is, $x_{j}=1$ for $j=1, \ldots, k$ and $x_{j}=0$ for $j=k+1, \ldots, n$. For players 
$j=1, \ldots, k$, we have $l_{j}-1 \geq k-1$; hence each finds it optimal to send a message given that $k-1$ other players do so as well. For players $j=k+1, \ldots, n$, we have $l_{j}-1<k$ since $k$ is defined such that $l_{k+1}<k+1$; hence each finds it optimal to not send a message given that $k$ other players do so. Therefore, $x$ is an equilibrium of $\Gamma^{c}(t)$.

Proof of Proposition 4.2. For all $j \in\{1, \ldots, n\}$,

$$
\begin{aligned}
\int_{T} u_{j}\left(X_{1}(t), \ldots,\right. & \left.X_{n}(t) ; c, t\right) d \gamma(t) \\
& \geq \int_{T} u_{j}\left(X_{1}(t), \ldots, X_{j-1}(t), X_{j}^{\prime}(t), X_{j+1}(t), \ldots, X_{n}(t) ; c, t\right) d \gamma(t)
\end{aligned}
$$

for every $X_{j}^{\prime} \in \mathcal{B}$ if and only if, for $\gamma$-a.e. $t \in T, X_{j}(t)$ solves

$$
\max _{x_{j} \in\{0,1\}} u_{j}\left(X_{1}(t), \ldots, X_{j-1}(t), x_{j}, X_{j+1}(t), \ldots, X_{n}(t) ; c, t\right) .
$$

Proof of Corollary 4.1. Let $c \geq 0$. For all $t \in T$, let $\mathcal{E}^{c}(t) \subset\{0,1\}^{n}$ be the set of equilibria for $\Gamma^{c}(t)$. By Proposition $4.1, \mathcal{E}^{c}(t)$ is non-empty. Since the payoffs in $\Gamma^{c}(t)$ depend continuously on $t$, the graph of the correspondence $\mathcal{E}^{c}: T \rightarrow\{0,1\}^{n}$ is closed. Therefore, $\mathcal{E}^{c}$ has a measurable selection $\left\langle X_{1}(\cdot), \ldots, X_{n}(\cdot)\right\rangle$. By Proposition $4.2,\left\langle X_{1}, \ldots, X_{n}\right\rangle$ is an equilibrium.

Proof of Proposition 4.3. Let $j \in\{1, \ldots, n\}$, let $c \geq 0$, and let $X_{-j} \equiv\left\langle X_{1}, \ldots, X_{j-1}\right.$, $\left.X_{j+1}, \ldots, X_{n}\right\rangle \in \mathcal{B}^{n-1}$ be a profile of strategies for senders other than $j$. Let $X_{j}^{-}$(resp., $X_{j}^{+}$) be the set of types $t$ for whom $j$ has a strict (resp., weak) incentive to send a message, given $c$ and $X_{-j}(t)$. That is,

$$
\begin{aligned}
& X_{j}^{-} \equiv\left\{t \in T \mid s_{j} t_{j}\left(\min \left\{1, m /\left(\# X_{-j}(t)+1\right)\right\}\right)>c\right\} \\
& X_{j}^{+} \equiv\left\{t \in T \mid s_{j} t_{j}\left(\min \left\{1, m /\left(\# X_{-j}(t)+1\right)\right\}\right) \geq c\right\} .
\end{aligned}
$$

Then $X_{j}^{-}$and $X_{j}^{+}$are both best responses. Furthermore, for any best response $X_{j} \in \mathcal{B}$, $X_{j}^{-} \underset{\text { a.s. }}{\subset} X_{j} \underset{\text { a.s. }}{\subset} X_{j}^{+}$(where $B \underset{\text { a.s. }}{\subset} B^{\prime}$ means that $\gamma$-a.e. element of $B$ is in $B^{\prime}$ ). The symmetric difference between $X_{j}^{-}$and $X_{j}^{+}$is

$$
\begin{aligned}
X_{j}^{+} \backslash X_{j}^{-} & =\left\{t \in T \mid s_{j} t_{j}\left(\min \left\{1, m /\left(\# X_{-j}(t)+1\right)\right\}\right)=c\right\} \\
& \subset \bigcup_{l=m}^{n}\left\{t \in T \mid t_{j}=(l / m)\left(c / s_{j}\right)\right\} .
\end{aligned}
$$

Assumption 4.1 implies that $\gamma\left\{t \in T \mid t_{j}=(l / m)\left(c / s_{j}\right)\right\}=0$ for $l=m, \ldots, n$, so $\gamma\left(X_{j}^{+} \backslash\right.$ $\left.X_{j}^{-}\right)=0$. Hence $X_{j}^{-}$and $X_{j}^{+}$, and any other best response $X_{j}$, are equivalent.

Lemma B.1 and Corollary B.1 are used in the proofs of Propositions 5.1 and 7.2. Recall that, for $l \in\{1, \ldots, n\}$ and $t \in T, v^{l}(t)$ is the $l$ th-highest valuation $s_{j} t_{j}$ of $t$ 's attention.

Lemma B.1 Let $p \geq 0$ and $t \in T$. Then $Y^{c}(t)$ is an equilibrium of $\Gamma^{c+p}(t)$ if and only if

$$
\begin{aligned}
v^{\# Y^{c}(t)}(t)-(c+p) & \geq 0, \\
\frac{m}{m+1} v^{m+1}(t)-(c+p) & \leq 0 .
\end{aligned}
$$


Proof. Equation (4) means that the lowest valuation sender who should send to type $t$ according to $Y^{c}(t)$ finds it profitable to do so, given that his message will be processed for sure. Equation (5) means that the highest valuation sender who should not be sending to type $t$ according to $Y^{c}(t)$ does not find it profitable to do so, given that $m$ senders are already sending messages. These two conditions are necessary and sufficient when $\# Y^{c}(t)=m$. If instead $\# Y^{c}(t)<m$, then $v^{m}(t) \leq c$ and so equation (5) is trivially satisfied while equation (4) is necessary and sufficient.

Corollary B.1 Let $t \in T$. Then $Y^{c}(t)$ is an equilibrium for $\Gamma^{c}(t)$ if and only if $\frac{m}{m+1} v^{m+1}(t) \leq$ $c$.

Proof. Consider Lemma B.1 for $p=0$. Equation (4) becomes $v^{\# Y^{c}(t)}(t)-c \geq 0$; this holds because of the efficiency of $Y^{c}$. The necessary and sufficient condition is thus equation (5), which becomes $\frac{m}{m+1} v^{m+1}(t) \leq c$.

Proof of Proposition 5.1. Part (1). Let $X$ be an equilibrium if $\Gamma^{c}$. We show that for $\gamma$-a.e. $t \in T, \# X(t) \geq \# Y^{c}(t)$. Let $t$ be such that $\left\langle X_{1}(t), \ldots, X_{n}(t)\right\rangle$ is an equilibrium for $\Gamma^{c}(t)$; this holds $\gamma$-a.e. according to Proposition 4.2. If at least $m$ messages are sent in this equilibrium (i.e., if $\# X(t) \geq m$ ), then $\# X(t) \geq \# Y^{c}(t)$ since $\# Y^{c}(t) \leq m$ (receivers are never overloaded in the efficient strategy profile). Suppose instead that $\# X(t)<m$. Then each sender's message is processed for sure, as would also be the case if one more sender sent a message. Assume that $s_{j} t_{j} \neq c$ for each $j$, which holds for $\gamma$-a.e. $t \in T$. Then sender $j$ sends a message in this equilibrium if and only if $s_{j} t_{j}>c$. This is true also for the efficient strategy profile when this inequality holds for at most $m$ senders. Hence, $\# X(t)=\# Y^{c}(t)$.

Part (2). Let $t \in Y_{j}^{c}$. Then (a) \# $\# Y_{-j}^{c}(t) \leq m-1$ and (b) $s_{j} t_{j}>c$. Condition (a) implies that $j$ 's message to $t$ would certainly be processed if sent; condition (b) implies that $j$ 's best response is therefore to send $t$ a message. Hence, $t \in X_{j}^{*}\left(Y_{-j}^{c} ; c\right)$.

Part (3). This follows directly from Corollary B.1.

Proof of Proposition 7.1. Suppose $Y^{c}$ is not an equilibrium of $\Gamma^{c}$. Let $p>0$. There is a sender $j$ for which $s_{j}>c$, since otherwise $Y^{c}$ is a profile of empty sets and is an equilibrium for $\Gamma^{c}$. Then define

$$
U \equiv\left\{t \in T \mid p>v_{j}(t)-c>0 \text { and } v_{j}(t)>v^{m+1}(t)\right\}
$$

The set $U$ consists of types $t \in T$ such that $Y^{c}(t)$ is not an equilibrium of $\Gamma^{c+p}(t)$ : the conditions $v_{j}(t)-c>0$ and $v_{j}(t)>v^{m+1}(t)$ mean that $t \in Y_{j}^{c}$, but the condition $v_{j}(t)-$ $(c+p)<0$ means that $j$ 's dominant strategy in $\Gamma^{c+p}(t)$ is to not send a message. Observe that $U$ is open because $v_{j}$ and $v^{m+1}$ are continuous. Hence, if we can show that $U$ intersects $\operatorname{supp}(\gamma)$, then $\gamma U>0$ and $Y^{c}$ is not an equilibrium of $\Gamma^{c+p}$.

Since $s_{j}>c$, there exists a $t_{j}^{\prime} \in(0,1)$ such that $p>s_{j} t_{j}^{\prime}-c>0$. The final step is to construct, from $t_{j}^{\prime}$, an element $t \in U \cap \operatorname{supp}(\gamma)$; we treat separately the two cases stated in the proposition. (1) Suppose $\operatorname{supp}(\gamma)=T$. Then $\left\langle 0, \ldots, t_{j}^{\prime}, \ldots, 0\right\rangle \in U \cap \operatorname{supp}(\gamma)$. (2) Suppose instead $\Delta^{n-1} \subset \operatorname{supp}(\gamma)$. If $m>1$, then choose any $i \neq j$. If instead there is $i$ such that $s_{i} \leq c$, then let $t$ be the element of $\Delta^{n-1}$ such that $t_{j}=t_{j}^{\prime}$ and $t_{i}=1-t_{j}^{\prime}$. Then $t \in U \cap \Delta^{n-1}$.

Proof of Proposition 7.2. Various steps are stated as lemmas. The assumptions (i) $\operatorname{supp}(\gamma)=\Delta^{n-1}$ and (ii) $m=1$ are maintained throughout. Let $v_{\max }^{2}=\max _{t \in \Delta^{n-1}} v^{2}(t)$ and $v_{\min }^{1}=\min _{t \in \Delta^{n-1}} v^{1}(t)$. 
Lemma B.2 Suppose $Y^{c}$ is not an equilibrium of $\Gamma^{c}$. Then $v_{\min }^{1} \geq(1 / 2) v_{\max }^{2}$ is necessary and sufficient for there to be some $p>0$ such that $Y^{c}$ is an equilibrium of $\Gamma^{c+p}$.

Proof of Lemma B.2. Suppose $Y^{c}$ is not an equilibrium of $\Gamma^{c}$. It follows from Corollary B.1 that $(1 / 2) v^{2}(t)>c$ for some $t \in \Delta^{n-1}$ and hence that $(1 / 2) v_{\max }^{2}>c$.

Suppose that $v_{\min }^{1} \geq(1 / 2) v_{\max }^{2}$. Let $p=(1 / 2) v_{\max }^{2}-c$, so $p>0$. Then $v_{\min }^{1}-(c+p) \geq 0$ and hence $v^{1}(t)-(c+p) \geq 0$ for all $t \in \Delta^{n-1}$. Furthermore, $(1 / 2) v_{\max }^{2}-(c+p)=0$ and hence $(1 / 2) v^{2}(t)-(c+p) \leq 0$ for all $t \in \Delta^{n-1}$. It follows from Lemma B.1 that $Y^{c}$ is an equilibrium of $\Gamma^{c+p}$.

Suppose instead that $v_{\min }^{1}<(1 / 2) v_{\max }^{2}$. We consider two cases: $0<p<(1 / 2) v_{\max }^{2}-c$ and $p \geq(1 / 2) v_{\max }^{2}-c$. Consider the first case. Let $U \equiv\left\{t \in T \mid(1 / 2) v^{2}(t)-(c+p)>0\right\}$, which is a set of types for which $Y^{c}(t)$ is not an equilibrium (from Lemma B.1). Since $v^{2}$ is continuous, $U$ is open; since $(1 / 2) v_{\max }^{2}-(c+p)>0$, we have $U \cap \Delta^{n-1} \neq \varnothing$. Hence, $\gamma U>0$ and $Y^{c}$ is not an equilibrium of $\Gamma^{c+p}$.

Let instead $p \geq(1 / 2) v_{\max }^{2}-c$, so that $v_{\min }^{1}-(c+p)<0$. Let $U=\left\{t \in T \mid p>v^{1}(t)-c>0\right\}$, which again is a set of types such that $Y^{c}(t)$ is not an equilibrium of $\Gamma^{c+p}(t)$. (The condition $v^{1}(t)-c>0$ means that a highest valuation sender should send to $t$ according to $Y^{c}(t)$, but the condition $v^{1}(t)-(c+p)<0$ means that every sender's dominant strategy in $\Gamma^{c+p}(t)$ is to not send a message.) Then $U \cap \Delta^{n-1} \neq \varnothing$ because (a) $v^{1}$ is continuous, (b) $v_{\min }^{1}<c+p$, (c) $v_{\max }^{2}>c$ (hence there is a $t \in \Delta^{n-1}$ such that $v^{1}(t)>c$ ), and (d) $\Delta^{n-1}$ is connected. Continuity of $v^{1}$ also implies that $U$ is open. Hence, $\gamma U>0$ and $Y^{c}$ is not an equilibrium of $\Gamma^{c+p}$.

Lemma B.3 Renumber the senders if necessary so that $s_{1} \geq \cdots \geq s_{n}$. Then

$$
\begin{aligned}
& v_{\min }^{1}=\left(\sum_{k=1}^{n} s_{k}^{-1}\right)^{-1}, \\
& v_{\max }^{2}=\left(s_{1}^{-1}+s_{2}^{-1}\right)^{-1} .
\end{aligned}
$$

Proof. The $t$ that minimizes $v^{1}(t)$ on $\Delta^{n-1}$ is such that $t_{1} s_{1}=\cdots=t_{n} s_{n}$, which means that $t_{j}=\left(1 / s_{j}\right) /\left(\sum_{k=1}^{n} s_{k}^{-1}\right)$ and hence $v^{1}(t)=s_{j} t_{j}=\left(\sum_{k=1}^{n} s_{k}^{-1}\right)^{-1}$.

The $t$ that maximizes $v^{2}(t)$ on $\Delta^{n-1}$ has positive values only for senders 1 and 2 , who have the two highest values of $s_{j} ; t$ is then such that $t_{1} s_{1}=t_{2} s_{2}$. Hence, $t_{1}=\left(1 / s_{1}\right) /\left(1 / s_{1}+1 / s_{2}\right)$ and $v^{2}(t)=\left(s_{1}^{-1}+s_{2}^{-1}\right)^{-1}$.

Lemma B.4 $v_{\min }^{1} \geq(1 / 2) v_{\max }^{2}$ if and only if condition (1), (2), or (3) of Proposition 7.2 holds.

Proof. Renumber the senders if necessary so that $s_{1} \geq \cdots \geq s_{n}$. Then the inequality $v_{\min }^{1} \geq(1 / 2) v_{\max }^{2}$ can be written as

$$
\begin{aligned}
& \sum_{k=1}^{n} \frac{1}{s_{k}} \leq 2\left(\frac{1}{s_{1}}+\frac{1}{s_{2}}\right), \\
& \sum_{k=3}^{n} \frac{1}{s_{k}} \leq \frac{1}{s_{1}}+\frac{1}{s_{2}} .
\end{aligned}
$$

If $n=2$, then equation (10) imposes no restriction of all. If $n=3$, then equation (10) becomes $s_{3}^{-1} \leq s_{1}^{-1}+s_{2}^{-1}$ (which in turn implies $s_{i}^{-1} \leq s_{j}^{-1}+s_{k}^{-1}$ for other permutations 
$\{i, j, k\}$ of $\{1,2,3\})$. If $n=4$, then equation (10) becomes $s_{3}^{-1}+s_{4}^{-1} \leq s_{1}^{-1}+s_{2}^{-1}$. Since $s_{1} \geq s_{2} \geq s_{3} \geq s_{4}$, this holds if and only if $s_{1}=s_{2}=s_{3}=s_{4}$.

Suppose $n>4$. Then the left-hand side of equation (10) is the sum of three or more terms, each of which is larger than the two terms summed on the right-hand side. Hence, the left-hand side is necessarily larger than the right-hand side.

This concludes the proof of Proposition 7.2.

Proof of Proposition 8.1. Part (1). First we show that these functions are well-defined; that is, we show that there is an equilibrium with the highest sales, one with the lowest sales, one with the highest profit, and one with the lowest profit. Let $c \geq 0$. From the proof of Corollary 4.1, recall that the graph of the equilibrium correspondence $\mathcal{E}^{c}: T \rightarrow\{0,1\}^{n}$ for the single-receiver games is closed. In particular, for each strategy profile of the singlereceiver games, the set of types for which it is an equilibrium is closed. Consider $\Sigma_{j}^{+}$. We can rank the single-receiver strategy profiles $\{0,1\}^{n}$ so that $j$ 's sales are weakly decreasing in the ranking for any single receiver game. (Profiles in which $j$ does not send a message get the lowest ranking; other profiles are ranked inversely by the number of other senders who send a message.) For each receiver type, we select the highest-ranked equilibrium strategy profile. This selection is measurable and hence defines an equilibrium for $\Gamma^{c}$. Sender $j$ 's sales in this equilibrium are as high as for any other equilibrium. The proofs for $\Sigma_{j}^{-}, \Pi_{j}^{+}$, and $\Pi_{j}^{-}$are analogous.

Next we show continuity. Fix $c \geq 0$. For $\delta>0$, let $\Theta^{\delta}$ be the set of types $t \in T$ such that $\Gamma^{c^{\prime}}(t)$ and $\Gamma^{c}(t)$ have the same equilibria for any $c^{\prime}$ such that $\left|c-c^{\prime}\right|<\delta$. Let $\epsilon>0$. We show later that there is a $\delta>0$ such that $\gamma\left(T \backslash \Theta^{\delta}\right)<\epsilon$. If $\left|c-c^{\prime}\right|<\delta$, then equilibria with the highest sales for a sender $j$ in the games $\Gamma^{c}$ and $\Gamma^{c^{\prime}}$ can differ only with respect to types in $T \backslash \Theta^{\delta}$; hence, $\left|\Sigma_{j}^{+}(c)-\Sigma_{j}^{+}\left(c^{\prime}\right)\right|<\epsilon$. Therefore, $\Sigma_{j}^{+}$is continuous. The proofs for $\Sigma_{j}^{-}, \Pi_{j}^{+}$, and $\Pi_{j}^{-}$are analogous.

We still have to show the existence of a $\delta>0$ such that $\gamma\left(T \backslash \Theta^{\delta}\right)<\epsilon$. Consider a type $t \in T$ such that for all $j \in\{1, \ldots, n\}$ and $l \in\{m, \ldots, n\}, s_{j} t_{j}(m / l) \neq c$; Assumption 4.1 implies that the set of such types has full measure. Each of the equilibria in $\Gamma^{c}(t)$ is strict and persists for a small perturbation of $\delta$. Hence, $\gamma$-a.e. $t \in T$ belongs to $\Theta^{\delta}$ for some $\delta>0$. Using the countable additivity of $\gamma$, we then have that $\gamma \Theta^{\delta} \uparrow \gamma T$ as $\delta \downarrow 0$. Hence, for every $\epsilon>0$ there is some $\delta>0$ such that $\gamma\left(T \backslash \Theta^{\delta}\right)<\epsilon$.

Part (2). Game $\Gamma^{0}$ has a unique equilibrium in which senders target all receivers. Thus, $\Sigma_{j}^{+}(0)=\Sigma_{j}^{-}(0)$ and $\Pi_{j}^{+}(0)=\Pi_{j}^{-}(0)$.

Part (3). We construct the lower bound $B_{1}: \mathbb{R}_{+} \rightarrow \mathbb{R}$ on $\Sigma_{1}^{-}$. By a change of indices, the result applies also to senders $j \neq 1$.

Let $c \in \mathbb{R}_{+}$. Denote by $\tilde{X}_{1}$ the set of types to whom it is a strictly dominant strategy for sender 1 to send a message; for each sender $j \neq 1$, denote by $\tilde{X}_{j}$ the types to whom $j$ would not send a message if all other senders do so. That is,

$$
\begin{aligned}
& \tilde{X}_{1}=\left\{t \in T \mid(m / n) s_{1} t_{1}>c\right\}, \\
& \hat{X}_{j}=\left\{t \in T \mid(m / n) s_{j} t_{j}<c\right\} .
\end{aligned}
$$

In any equilibrium, player 1 sends a message to at least the types in $\tilde{X}_{1}$ and these types receive at most $n$ messages. For types in $\tilde{X}_{1} \cap\left(\bigcup_{j=2}^{n} \hat{X}_{j}\right)$, not all senders can send messages in equilibrium, so these types receive at most $n-1$ messages. Therefore, as a lower bound on $\Sigma_{1}^{-}(c)$, we have the sales $(m / n) \int_{T} t_{1} d \gamma$ obtained when all senders target all receivers, with two adjustments: types in $\bigcup_{j>1} \hat{X}_{j}$ are targeted by at most $n-1$ rather than $n$ senders; 
and types in $\tilde{X}_{1}^{\circ}$ (the complement of $\tilde{X}_{1}$ ) might not be targeted by any sender 1 - yet we have so far counted sales of up to $(m /(n-1)) t_{1}$ for such types. We thus have

$$
\sigma_{1}(X) \geq \frac{m}{n} \int_{T} t_{1} d \gamma+\left(\frac{m}{n-1}-\frac{m}{n}\right) \int_{\bigcup_{j=2}^{n} \hat{X}_{j}} t_{1} d \gamma-\frac{m}{n-1} \int_{\tilde{X}_{1}^{\circ}} t_{1} d \gamma
$$

We obtain another lower bound by breaking up the integral $\int_{\bigcup_{j=2}^{n}} \hat{X}_{j} t_{1} d \gamma$ into one integral for each $\hat{X}_{j}$ and then subtracting potential overlap, replacing $t_{1}$ by its upper bound 1 as we do so:

$$
\begin{aligned}
\Sigma_{1}^{-}(c) \geq \frac{m}{n} \int_{T} t_{1} d \gamma & +\left(\frac{m}{n-1}-\frac{m}{n}\right) \sum_{j=2}^{n} \int_{\hat{X}_{j}} t_{1} d \gamma \\
& -\left(\frac{m}{n-1}-\frac{m}{n}\right)\left(\sum_{j=2}^{n} \sum_{\substack{i \geq 2 \\
i \neq j}} \gamma\left(\hat{X}_{j} \cap \hat{X}_{i}\right)\right)-\frac{m}{n-1} \int_{\tilde{X}_{1}^{\circ}} t_{1} d \gamma
\end{aligned}
$$

The double summation does not appear if $n=2$. Otherwise, observe that $\hat{X}_{j} \cap \hat{X}_{i}=$ $\left\{t \in T \mid t_{i}<(c n) /\left(m s_{i}\right) \& t_{j}<(c n) /\left(m s_{j}\right)\right\}$. Therefore, according to Assumption 8.1, $\gamma\left(\hat{X}_{j} \cap\right.$ $\left.\hat{X}_{i}\right) \leq \bar{k} c^{2}$, where $\bar{k}=k\left(n /\left(m \max \left\{s_{j} \mid j \neq 1\right\}\right)\right)^{2}$ and $k$ is the constant given in part 3 of Assumption 8.1. We rewrite the integrals in equation (11) using the notation and assumptions in parts 1 and 2 of Assumption 8.1. For example, since $\hat{X}_{j}=\left\{t \in T \mid(m / n) s_{j} t_{j}<c\right\}$, we have $\int_{\hat{X}_{j}} t_{1} d \gamma=\int_{0}^{c n / m s_{j}} E\left[t_{1} \mid t_{j}\right] f_{j}\left(t_{j}\right) d t_{j}$. We thereby obtain the following lower bound on $\Sigma_{1}^{-}(c)$ :

$$
\begin{aligned}
B_{1}(c) \equiv \frac{m}{n} E\left[t_{1}\right]+ & \left(\frac{m}{n-1}-\frac{m}{n}\right) \sum_{j=2}^{n} \int_{0}^{c n / m s_{j}} E\left[t_{1} \mid t_{j}\right] f_{j}\left(t_{j}\right) d t_{j} \\
& -\left(\frac{m}{n-1}-\frac{m}{n}\right)(n-1)(n-2) \bar{k} c^{2}-\frac{m}{n-1} \int_{0}^{c n / m s_{1}} t_{1} f_{1}\left(t_{1}\right) d t_{1}
\end{aligned}
$$

Note that $B_{1}$ is continuously differentiable. In particular, $t_{j} \mapsto E\left[t_{1} \mid t_{j}\right] f_{j}\left(t_{j}\right)$ is continuous because (a) $f_{j}$ is continuous and (b) $E\left[t_{1} \mid t_{j}\right]$ is continuous when $f_{j}>0$. The derivative of $B_{1}$ evaluated at $c=0$ is

$$
\begin{aligned}
\left.\frac{d}{d c} B_{1}(c)\right|_{c=0} & =\left.\left(\frac{m}{n-1}-\frac{m}{n}\right) \sum_{j=2}^{n} \frac{n}{m s_{j}} E\left[t_{1} \mid t_{j}=c n / m s_{j}\right] f_{j}\left(c n / m s_{j}\right)\right|_{c=0} \\
& =\frac{1}{n-1} \sum_{j=2}^{n} s_{j}^{-1} E\left[t_{1} \mid t_{j}=0\right] f_{j}(0) .
\end{aligned}
$$

This derivative is strictly positive if there is an $i \neq j$ such that $f_{i}(0)>0$ and $E\left[t_{j} \mid t_{i}=0\right]>$ 0 , so that $B_{1}(\cdot)$ is strictly increasing in a neighborhood of zero.

Finally, observe that $B_{1}(0)=\Sigma_{1}^{-}(0)$. Given the properties of $\Sigma_{1}^{+}$and $\Sigma_{1}^{-}$, these must be strictly increasing in a neighborhood of zero if $B_{1}$ is.

Part (4). Let $\hat{B}_{1}(c)=s_{1} B_{1}(c)-c$. Then $\hat{B}_{1}(c)$ is a lower bound on $\Pi_{1}^{-}$because $B_{1}(c)$ is a lower bound on $\Sigma_{1}^{-}(c)$. The function $\hat{B}_{1}(\cdot)$ is continuously differentiable and

$$
\left.\frac{d}{d c} \hat{B}_{1}(c)\right|_{c=0}=s_{1} \frac{1}{n-1} \sum_{j=2}^{n} s_{j}^{-1} E\left[t_{1} \mid t_{j}=0\right] f_{j}(0)-1
$$


Equation (2) implies that $\left.\frac{d}{d c} \hat{B}_{1}(\cdot)\right|_{c=0}>0$, so that $\hat{B}_{1}(\cdot)$ is also strictly increasing in a neighborhood of zero.

We also see that $\hat{B}_{1}(0)=\Pi_{1}^{-}(0)$. Given the properties of $\Pi_{1}^{+}$and $\Pi_{1}^{-}$, these must be strictly increasing in a neighborhood of zero if $\hat{B}_{1}$ is.

\section{References}

Butters, G. R. (1977). Equilibrium distributions of sales and advertising prices. Review of Economic Studies, 44, 465-491.

Denning, P. J. (1982). Electronic junk. Communications of the ACM, 25, 163-165.

Diamond, P. (1971). A model of price adjustment. Journal of Economic Theory, 3, 156-168.

Grether, D., Schwartz, A., and Wilde, L. (1986). The irrelevance of information overload: An analysis of search and disclosure. Southern California Law Review, 59, 277-303.

Grossman, G. M. and Shapiro, C. (1984). Informative advertising and differentiated products. Review of Economic Studies, 51, 63-81.

Hiltz, S. and Turoff, M. (1985). Structuring computer-mediated communication systems to avoid information overload. Communications of the ACM, 28, 680-689.

Jacoby, J., Speller, D. E., and Kohn, C. A. (1974). Brand choice behavior as a function of information load. Journal of Marketing Research, 11, 63-69.

Keller, K. L. and Staelin, R. (1987). Effects of quality and quantity of information on decision effectiveness. Journal of Consumer Research, 14, 200-213.

Kraut, R. E., Sunder, S., Morris, J., Filer, D., and Cronin, M. (2000). Markets for attention: Can email postage save us from spam? Carnegie Mellon University and Yale University.

Lai, K.-Y., Malone, T. W., and Yu, K.-C. (1988). Object lens: A spreadsheet for cooperative work. ACM Transactions on Office Information Systems, 6, 332-353.

Libowski, Z. (1975). Sensory and information inputs overload: Behavioral effects. Comprehensive Psychiatry, 16, 199-221.

Malhotra, N. K. (1982). Information load and consumer decision making. Journal of Consumer Research, 8, 419-430.

Malone, T. W., Grant, K. R., Turbak, F. A., Brobst, S. A., and Cohen, M. D. (1987). Intelligent information-sharing systems. Communications of the ACM, 30, 390-402.

Meyer, R. J. and Johnson, E. J. (1989). Information overload and the nonrobustness of linear models: A comment on Keller and Staelin. Journal of Consumer Research, 15, 498-503.

Robert, J. and Stahl, D. O. (1993). Informative price advertising in a sequential search model. Econometrica, 61, 657-686.

Rosenthal, R. (1980). Equilibrium price dispersions. Review of Economic Studies, 52, 457504.

Satterthwaite, M. A. (1979). Consumer information, equilibrium industry price, and the number of sellers. Bell Journal of Economics, 10, 483-502. 
Schwartz, A. and Wilde, L. L. (1983). Imperfect information in markets for contract terms: The examples of warranties and security interests. Virginia Law Review, 69, 1387-1485.

Stahl, D. O. (1989). Oligopolistic pricing with sequential consumer search. American Economic Review, 79, 700-712.

Stahl, D. O. (1994). Oligopolist pricing and advertising. Journal of Economic Theory, 64, $162-177$.

Stegeman, M. (1991). Advertising in competitive markets. American Economic Review, 81, $210-223$.

Vickrey, W. (1961). Counterspeculation, auctions and competitive sealed tenders. Journal of Finance, 16, 8-37. 